\title{
ORLICZ CAPACITIES AND APPLICATIONS TO SOME EXISTENCE QUESTIONS FOR ELLIPTIC PDES HAVING MEASURE DATA
}

\author{
Alberto Fiorenza ${ }^{1}$ And Alain Prignet, ${ }^{2}$
}

\begin{abstract}
We study the sequence $u_{n}$, which is solution of $-\operatorname{div}\left(a\left(x, \nabla u_{n}\right)\right)+\Phi^{\prime \prime}\left(\left|u_{n}\right|\right) u_{n}=f_{n}+g_{n}$ in $\Omega$ an open bounded set of $\mathbf{R}^{N}$ and $u_{n}=0$ on $\partial \Omega$, when $f_{n}$ tends to a measure concentrated on a set of null Orlicz-capacity. We consider the relation between this capacity and the $N$-function $\Phi$, and prove a non-existence result.
\end{abstract}

Mathematics Subject Classification. 35J60, 46E30, 31C45.

Received June 27, 2002. Revised January 17, 2003.

\section{INTRODUCTION}

Let $\Omega$ be a bounded open subset of $\mathbf{R}^{N}, N>2$, we study the non-existence of a solution for the following nonlinear elliptic problem (that is our model problem)

$$
\left\{\begin{array}{cl}
-\Delta u+|u|^{q-1} u=\mu & \text { in } \Omega \\
u=0 & \text { on } \partial \Omega
\end{array}\right.
$$

in the following sense: let $f_{n}$ be a sequence of smooth functions that tends to a measure $\mu$ in a sense that we will precise. Let $u_{n}$ be the sequence of solutions of

$$
\left\{\begin{array}{cl}
-\Delta u_{n}+\left|u_{n}\right|^{q-1} u_{n}=f_{n} & \text { in } \Omega, \\
u_{n}=0 & \text { on } \partial \Omega,
\end{array}\right.
$$

we will consider the case, with respect to the measure $\mu$ and the value of $q$, where $u_{n}$ converges to a function $u$ that does not satisfy (1.1).

The first result was due to Brezis (see [9]). Let $\Omega$ be a bounded open subset of $\mathbf{R}^{N}, N>2$, with $0 \in \Omega$, let $f$ be a function in $L^{1}(\Omega)$, and let $f_{n}$ be a sequence of $L^{\infty}(\Omega)$ functions such that

$$
\lim _{n \rightarrow+\infty} \int_{\Omega \backslash B_{\rho}(0)}\left|f_{n}-f\right| \mathrm{d} x=0, \quad \forall \rho>0 .
$$

Keywords and phrases. Elliptic equation, Orlicz space, measure, capacity.

${ }^{1}$ Dipartimento di Costruzioni e Metodi Matematici in Architettura, Università di Napoli, via Monteoliveto 3, 80134 Napoli, Italy, and Istituto per le Applicazioni del Calcolo "Mauro Picone", Sezione di Napoli, Consiglio Nazionale delle Ricerche, via Pietro Castellino 111, 80131 Napoli, Italy; e-mail: fiorenza@unina.it

2 Mathématiques, Université d'Orléans, rue de Chartres, 45067 Orléans Cedex 2, France;

e-mail: prignet@labomath.univ-orleans.fr 
Let $u_{n}$ be the sequence of solutions of (1.2) with $q \geq \frac{N}{N-2}$. Then $u_{n}$ converges to the unique solution $u$ of the equation $-\Delta u+|u|^{q-1} u=f$.

If $f=0$, an example of functions $f_{n}$ satisfying condition (1.3) is that of a sequence of nonnegative functions in $L^{\infty}(\Omega)$ converging in the weak* topology of measures to $\delta_{0}$, the Dirac mass concentrated at the origin. In this case, $u_{n}$ converges to zero. The result of [9] is strongly connected with a theorem by Bénilan and Brezis (see [9]), which states that the problem $-\Delta u+|u|^{q-1} u=\delta_{0}$ has no distributional solution if $q \geq \frac{N}{N-2}$. On the other hand (see [7] and [9]), if $q<\frac{N}{N-2}$, then there exists a unique solution of

$$
\left\{\begin{array}{cl}
-\Delta u+|u|^{q-1} u=\delta_{0} & \text { in } \Omega \\
u=0 & \text { on } \partial \Omega .
\end{array}\right.
$$

Thus the preceding theorem can be seen as a nonexistence result for this problem, in the sense that if one looks for solutions obtained by approximation of (1.4), then one does not find a "reasonable" solution (that is stable with respect to the right-hand side).

The "dividing range" $\frac{N}{N-2}$ basically depends on two facts: the linearity of the laplacian operator (i.e., the dependence of order 1 with respect to the gradient of $u$ ), and the fact that the Dirac $\delta_{0}$ is a measure which is concentrated on a point: a set of zero $N$-capacity (see definition below). In the case $q \geq \frac{N}{N-2}$, which is equivalent to $2 q^{\prime} \leq N, \delta_{0}$ is not "absolutely continuous" with respect to the $N$-capacity and hence also to the $2 q^{\prime}$-capacity and there is no solution of (1.4). If $q<\frac{N}{N-2}$, which is equivalent to $2 q^{\prime}>N, \delta_{0}$ is "absolutely continuous" with respect to the $2 q^{\prime}$-capacity and there is a solution of (1.4).

This fact is strictly related to the result of [14], where a necessary and sufficient condition for the existence of a solution is given. More precisely, the equation

$$
\left\{\begin{array}{cl}
-\Delta u+|u|^{q-1} u=\mu & \text { in } \Omega \\
u=0 & \text { on } \partial \Omega
\end{array}\right.
$$

has a solution if and only if $\mu$ belongs to $L^{1}(\Omega)+W^{-2, q}(\Omega)$. If $\mu$ is a measure that is "absolutely continuous" with respect to the $\left(2, q^{\prime}\right)$-capacity, which is defined in Definition 2.5 , then $\mu$ belongs to $L^{1}(\Omega)+W^{-2, q}(\Omega)$ and (1.5) has a solution. Moreover in [5], the singularities for (1.5) are removable (which is a notion that can be seen closed to the result of non existence) if and only if $\mu$ is "absolutely continuous" with respect to the $\left(2, q^{\prime}\right)$-capacity.

In order to point out the relations between these results and capacities, we recall that we have (according to the Gagliardo-Nirenberg inequalities)

$$
\operatorname{cap}_{2, q^{\prime}}(E)=0 \quad \Longrightarrow \quad \operatorname{cap}_{1,2 q^{\prime}}(E)=0,
$$

and that, by [1] (Th. 5.5.1), we have, for every set $E$,

$$
\operatorname{cap}_{1,2 q^{\prime}+\varepsilon}(E)=0 \quad \Longrightarrow \quad \operatorname{cap}_{2, q^{\prime}}(E)=0, \quad \forall \varepsilon>0 .
$$

The result of [9] has been extended to nonlinear operators of Leray-Lions type (see definition below) and measures concentrated on sets of null $r$-capacities in [23]:

Theorem 1.1. Let $1<p<r \leq N$, a satisfying (2.7-2.9) and let $\lambda=\lambda^{+}-\lambda^{-}$be a bounded Radon measure concentrated on a set $E$ of zero $r$-capacity. Let $f_{n}=f_{n}^{\oplus}-f_{n}^{\ominus}$ (with $f_{n}^{\oplus}$ and $f_{n}^{\ominus}$ nonnegative functions) be a sequence of $L^{\infty}(\Omega)$ functions that converges to $\lambda$ in the sense

$$
\lim _{n \rightarrow+\infty} \int_{\Omega} f_{n}^{\oplus} \varphi \mathrm{d} x=\int_{\Omega} \varphi \mathrm{d} \lambda^{+}, \quad \lim _{n \rightarrow+\infty} \int_{\Omega} f_{n}^{\ominus} \varphi \mathrm{d} x=\int_{\Omega} \varphi \mathrm{d} \lambda^{-},
$$


for every function $\varphi$ which is continuous and bounded on $\Omega$. Let $g$ be a function in $L^{1}(\Omega)$, and let $g_{n}$ be a sequence of $L^{\infty}(\Omega)$ functions which converges to $g$ weakly in $L^{1}(\Omega)$. Let

$$
q>\frac{r(p-1)}{r-p}
$$

and let $u_{n}$ be the solution in $W_{0}^{1, p}(\Omega)$ of the problem

$$
\left\{\begin{array}{cl}
-\operatorname{div}\left(a\left(x, \nabla u_{n}\right)\right)+\left|u_{n}\right|^{q-1} u_{n}=f_{n}+g_{n} & \text { in } \Omega \\
u_{n}=0 & \text { on } \partial \Omega .
\end{array}\right.
$$

Then, as $n$ tends to infinity, $\left|\nabla u_{n}\right|^{p-1}$ converges strongly to $|\nabla u|^{p-1}$ in $L^{\sigma}(\Omega)$, for every $\sigma<\frac{p q}{(q+1)(p-1)}$, where $u$ is the unique entropy solution of

$$
\left\{\begin{array}{cl}
-\operatorname{div}(a(x, \nabla u))+|u|^{q-1} u=g & \text { in } \Omega \\
u=0 & \text { on } \partial \Omega .
\end{array}\right.
$$

Moreover,

$$
\lim _{n \rightarrow+\infty} \int_{\Omega}\left|u_{n}\right|^{q-1} u_{n} \varphi \mathrm{d} x=\int_{\Omega}|u|^{q-1} u \varphi \mathrm{d} x+\int_{\Omega} \varphi \mathrm{d} \lambda, \quad \forall \varphi \in C_{\mathrm{c}}^{0}(\Omega) .
$$

Remark 1.2. Since this theorem deals with rather general operators and measures, the concept of solution in the sense of distributions of problems like (1.6) may not be convenient in order to have uniqueness of solutions. Hence the notion of entropy solutions (see Def. 2.9) has been used.

In order to avoid the loss between $q \geq \frac{N}{N-2}$ (see [9]) and $q>\frac{N}{N-2}$ (see Th. 1.1 with $p=2$ and $r=N$ ) and between the $2 q^{\prime}$-capacity and the $\left(2, q^{\prime}\right)$-capacity, we will extend the result of [23] to low order terms more general that $|u|^{q-1} u$ in the context of Orlicz spaces. The best approach for this new context will involve also the notion of Orlicz capacity. Such a notion has been already introduced in literature (see [4]). In spite of this, we will adopt a new equivalent definition (see Def. 2.6), which is closer to the classical one used in the context of the Sobolev spaces.

\section{The MAIN RESUlts}

\subsection{Definitions}

First let us give the definitions useful to understand the results.

Definition 2.1. An $N$-function is a function $\Phi$ continuous on $[0, \infty[$, increasing, convex, and such that $\lim _{x \rightarrow 0} \Phi(x) / x=0, \lim _{x \rightarrow \infty} \Phi(x) / x=+\infty$. For our purposes we will assume also that $\Phi \in C^{1}([0, \infty[)$, $\Phi^{\prime}$ increasing, and

$$
c_{1} \min \left(s^{q_{1}-1}, s^{q_{2}-1}\right) \Phi^{\prime}(t) \leq \Phi^{\prime}(s t) \leq c_{2} \max \left(s^{q_{1}-1}, s^{q_{2}-1}\right) \Phi^{\prime}(t)
$$

for some $q_{1}, q_{2}>1$. 
Remark 2.2. Condition (2.1) means that the growth of $\Phi$ "lies between" that one of the powers $t^{q_{1}}, t^{q_{2}}$. Indeed it implies all the following inequalities:

$$
\begin{gathered}
c_{1} \min \left(s^{q_{1}}, s^{q_{2}}\right) \Phi(t) \leq \Phi(s t) \leq c_{2} \max \left(s^{q_{1}}, s^{q_{2}}\right) \Phi(t) \\
c_{1} \min \left(s^{\frac{q_{1}}{q_{1}-1}}, s^{\frac{q_{2}}{q_{2}-1}}\right) \widetilde{\Phi}(t) \leq \widetilde{\Phi}(s t) \leq c_{2} \max \left(s^{\frac{q_{1}}{q_{1}-1}}, s^{\frac{q_{2}}{q_{2}-1}}\right) \widetilde{\Phi}(t) \\
c_{1} \min \left(s^{\frac{1}{q_{1}}}, s^{\frac{1}{q_{2}}}\right) \Phi^{-1}(t) \leq \Phi^{-1}(s t) \leq c_{2} \max \left(s^{\frac{1}{q_{1}}}, s^{\frac{1}{q_{2}}}\right) \Phi^{-1}(t) \\
c_{1} \min \left(s^{\frac{q_{1}-1}{q_{1}}}, s^{\frac{q_{2}-1}{q_{2}}}\right) \widetilde{\Phi}^{-1}(t) \leq \widetilde{\Phi}^{-1}(s t) \leq c_{2} \max \left(s^{\frac{q_{1}-1}{q_{1}}}, s^{\frac{q_{2}-1}{q_{2}}}\right) \widetilde{\Phi}^{-1}(t) \\
c_{1} \min \left(s^{\frac{1}{q_{1}-1}}, s^{\frac{1}{q_{2}-1}}\right) \widetilde{\Phi}^{\prime}(t) \leq \widetilde{\Phi}^{\prime}(s t) \leq c_{2} \max \left(s^{\frac{1}{q_{1}-1}}, s^{\frac{1}{q_{2}-1}}\right) \widetilde{\Phi}^{\prime}(t)
\end{gathered}
$$

for all $s, t>0$. The constants $c_{1}, c_{2}$ need not to be the same in each line. We remark that the inequalities stated above are not equivalent; however, with the help of the arguments given in [24], it can be proved that (2.1) implies them all. Remark that (2.2) implies that $\Phi \in \Delta_{2}$.

Definition 2.3. The $N$-function $\Phi$ belongs to $\Delta_{2}$ if there exist $c>0$ and $t_{0} \geq 0$ such that

$$
\Phi(2 t) \leq c \Phi(t) \quad \forall t \geq t_{0} .
$$

Definition 2.4. The complementary function of $\Phi$, denoted by $\widetilde{\Phi}$, is defined by

$$
\widetilde{\Phi}(s)=\sup _{t \geq 0}[s t-\Phi(t)] \quad \forall s \geq 0 .
$$

It can be proved that if $\Phi$ is an $N$-function, also $\widetilde{\Phi}$ is an $N$-function. If $\Phi^{\prime}$ is strictly increasing, $(\widetilde{\Phi})^{\prime}(t)=$ $\left(\Phi^{\prime}\right)^{-1}(t) \forall t \geq 0$.

In the following $\Omega$ denotes an open bounded set of $\mathbf{R}^{N}$. For $\Phi$ satisfying the Definition 2.1, the Orlicz class $L^{\Phi}(\Omega)$ is defined by

$$
L^{\Phi}(\Omega)=\left\{f \in L_{\mathrm{loc}}^{1}(\Omega): \int_{\Omega} \Phi(|f|) \mathrm{d} x<+\infty\right\}
$$

The Orlicz class $L^{\Phi}(\Omega)$, equipped with the norm

$$
\|f\|_{\Phi}=\inf \left\{k>0: \int_{\Omega} \Phi\left(\frac{|f|}{k}\right) \mathrm{d} x \leq 1\right\}
$$

becomes the so-called Orlicz space, which is a reflexive Banach space whose dual is $L^{\widetilde{\Phi}}(\Omega)$. In the following we will assume that the reader is familiar with the Orlicz space theory, deeply studied (for instance) in [17, 19,25].

One can also define the Orlicz-Marcinkiewicz spaces by

$$
M^{\Phi}(\Omega)=\left\{f \in L_{\mathrm{loc}}^{1}(\Omega): t \mapsto \text { meas }\{f>t\} \Phi(t) \text { is bounded }\right\} .
$$

Definition 2.5. Let $0<\alpha<N$ and let $r$ be a real number, with $r>1$. Let $K$ be a compact subset of $\Omega$. The $(\alpha, r)$-capacity of $K$ with respect to $\Omega$ is defined as

$$
\operatorname{cap}_{\alpha, r}(K)=\operatorname{cap}_{\alpha, r}(K, \Omega)=\inf \left\{\|u\|_{W_{0}^{\alpha, r}(\Omega)}^{r}: u \in C_{\mathrm{c}}^{\infty}(\Omega), u \geq \chi_{K}\right\},
$$


where $\chi_{K}$ is the characteristic function of $K$; we will use the convention that $\inf \emptyset=+\infty$. The $(\alpha, r)$-capacity of any open subset $U$ of $\Omega$ is then defined by

$$
\operatorname{cap}_{\alpha, r}(U)=\operatorname{cap}_{\alpha, r}(U, \Omega)=\sup \left\{\operatorname{cap}_{\alpha, r}(K), K \text { compact, } K \subset U\right\},
$$

and the $(\alpha, r)$-capacity of any set $E \subset \Omega$ by

$$
\operatorname{cap}_{\alpha, r}(E)=\operatorname{cap}_{\alpha, r}(E, \Omega)=\inf \left\{\operatorname{cap}_{\alpha, r}(U), U \text { open, } E \subset U\right\} .
$$

We introduce now the following definition, which represents a generalization of the previous one. We will see in Section 4 that this formulation is equivalent to that one appearing in [4].

Definition 2.6. Let $K$ be a compact subset of $\Omega$. Let $A$ satisfying Definition 2.1. The $A$-capacity of $K$ with respect to $\Omega$ is defined as:

$$
\operatorname{cap}_{1, A}(K)=\inf \left\{A\left(\|\nabla u\|_{A}\right): u \in C_{\mathrm{c}}^{\infty}(\Omega), u \geq \chi_{K}\right\},
$$

where $\chi_{K}$ is the characteristic function of $K$; we will use the convention that $\inf \emptyset=+\infty$. The $A$-capacity of any open subset $U$ of $\Omega$ is then defined by:

$$
\operatorname{cap}_{1, A}(U)=\sup \left\{\operatorname{cap}_{1, A}(K), K \text { compact, } K \subset U\right\},
$$

and the $A$-capacity of any set $E \subset \Omega$ by

$$
\operatorname{cap}_{1, A}(B)=\inf \left\{\operatorname{cap}_{1, A}(U), U \text { open, } E \subset U\right\} .
$$

Let $p$ be a real number, with $1<p<N$, and let $p^{\prime}$ be its conjugate Hölder exponent (i.e., $1 / p+1 / p^{\prime}=1$ ). Let $a: \Omega \times \mathbf{R}^{N} \rightarrow \mathbf{R}^{N}$ be a Carathéodory function $\left(i . e ., a(\cdot, \xi)\right.$ is measurable on $\Omega$ for every $\xi$ in $\mathbf{R}^{N}$, and $a(x, \cdot)$ is continuous on $\mathbf{R}^{N}$ for almost every $x$ in $\Omega$ ), such that the following holds:

$$
\begin{gathered}
a(x, \xi) \cdot \xi \geq \alpha|\xi|^{p}, \\
|a(x, \xi)| \leq \beta\left[b(x)+|\xi|^{p-1}\right], \\
{[a(x, \xi)-a(x, \eta)] \cdot(\xi-\eta)>0,}
\end{gathered}
$$

for almost every $x$ in $\Omega$, for every $\xi, \eta$ in $\mathbf{R}^{N}$, with $\xi \neq \eta$, where $\alpha$ and $\beta$ are two positive constants, and $b$ is a nonnegative function in $L^{p^{\prime}}(\Omega)$.

Under assumptions $(2.7,2.8)$ and $(2.9), u \mapsto-\operatorname{div}(a(x, \nabla u))$ is a uniformly elliptic, coercive and pseudomonotone operator acting from $W_{0}^{1, p}(\Omega)$ to its dual $W^{-1, p^{\prime}}(\Omega)$, and so it is surjective (see [18]).

Let us denote $C(\Omega)$ the space of the real valued continuous functions on $\Omega$, equipped with the topology of uniform convergence on compact subsets of $\Omega$. If $K$ is compact, $C(K)$ is usually normed with the supremum norm $\|\cdot\|_{L^{\infty}(K)} \cdot C_{c}(\Omega)$ is the subset of $C(\Omega)$ consisting of functions with compact support contained in $\Omega$. The dual of the space $C_{c}(\Omega)$ is denoted by $\mathcal{M}(\Omega)$, the bounded measures on $\Omega$. The set of positive measures on $\Omega$, is denoted by $\mathcal{M}^{+}(\Omega)$. For $K$ compact, the symbol $\mathcal{M}^{+}(K)$ has analogous meaning; such space will be used mainly in some intermediate auxiliary statements in Section 4.

Let $\lambda$ be a bounded measure on $\Omega$. We say that $\lambda$ is concentrated on a set $E$ if $\lambda(B)=\lambda(B \cap E)$ for every Borelian subset $B$ of $\Omega$. Thanks to the Hahn decomposition theorem, given a signed Radon measure $\lambda$ on $\Omega$, we can decompose it as the difference of two nonnegative, mutually singular, measures:

$$
\lambda=\lambda^{+}-\lambda^{-} .
$$


If $\lambda$ is concentrated on a set $E$, as a consequence of the fact that $\lambda^{+}$and $\lambda^{-}$are mutually singular, we have that $\lambda^{+}$is concentrated on a set $E^{+}, \lambda^{-}$is concentrated on a set $E^{-}$, and $E^{+} \cap E^{-}=\emptyset$.

Definition 2.7. Let $\lambda$ be a measure, decomposed as $\lambda^{+}-\lambda^{-}$, and let be approximations $f_{n}$ of $\lambda$ made in the following way: $f_{n}=f_{n}^{\oplus}-f_{n}^{\ominus}$, where $\left\{f_{n}^{\oplus}\right\}$ and $\left\{f_{n}^{\ominus}\right\}$ are sequences of nonnegative $L^{\infty}(\Omega)$ functions such that

$$
\lim _{n \rightarrow+\infty} \int_{\Omega} f_{n}^{\oplus} \varphi \mathrm{d} x=\int_{\Omega} \varphi \mathrm{d} \lambda^{+}, \quad \lim _{n \rightarrow+\infty} \int_{\Omega} f_{n}^{\ominus} \varphi \mathrm{d} x=\int_{\Omega} \varphi \mathrm{d} \lambda^{-},
$$

for every function $\varphi$ which is continuous and bounded on $\Omega$.

We explicitly remark that $f_{n}^{\oplus}$ and $f_{n}^{\ominus}$ may not be the positive and negative parts of $f_{n}$ (that is to say, their supports may not be disjoint). Observe that choosing $\varphi \equiv 1$ in (2.10) we obtain

$$
\left\|f_{n}^{\oplus}\right\|_{L^{1}(\Omega)} \leq c, \quad\left\|f_{n}^{\ominus}\right\|_{L^{1}(\Omega)} \leq c .
$$

Since we will deal with right hand side which are some measures, the solution may not be in $L_{\text {loc }}^{1}(\Omega)$, thus there distributional gradient may not be defined. Thus we will use the following definition of "gradient".

Before this, we define, for $k>0$,

$$
T_{k}(s)=\max (-k, \min (k, s)), \quad \forall s \in \mathbf{R},
$$

the truncature at levels $\pm k$.

Definition 2.8. Let $u$ be a measurable function on $\Omega$ such that $T_{k}(u)$ belongs to $W_{0}^{1, p}(\Omega)$ for every $k>0$. Then (see [6], Lem. 2.1) there exists a unique measurable function $v: \Omega \rightarrow \mathbf{R}^{N}$ such that

$$
\nabla T_{k}(u)=v \chi_{\{|u| \leq k\}}, \quad \text { almost everywhere in } \Omega, \text { for every } k>0 .
$$

We will define the gradient of $u$ as the function $v$, and we will denote it by $v=\nabla u$. If $u$ belongs to $W_{0}^{1,1}(\Omega)$, then this gradient coincides with the usual gradient in distributional sense.

For nonlinear elliptic equations with right hand sides measures or $L^{1}(\Omega)$ functions, there is no uniqueness of distributional solutions, thus we will use the following notion of entropy solution (see [6]).

Definition 2.9. Let $a$ satisfy the assumptions above, let $g$ be a function in $L^{1}(\Omega)$ and let $\Phi \in C^{2}([0, \infty[)$ be a convex function. A measurable function $u$ such that $T_{k}(u)$ belongs to $W_{0}^{1, p}(\Omega)$ for every $k>0$ is an entropy solution of the equation

$$
\left\{\begin{array}{cl}
-\operatorname{div}(a(x, \nabla u))+\Phi^{\prime \prime}(|u|) u=g & \text { in } \Omega, \\
u=0 & \text { on } \partial \Omega,
\end{array}\right.
$$

if $\Phi^{\prime \prime}(|u|)|u|$ belongs to $L^{1}(\Omega)$, and

$$
\int_{\Omega} a(x, \nabla u) \cdot \nabla T_{k}(u-\varphi) \mathrm{d} x+\int_{\Omega} \Phi^{\prime \prime}(|u|) u T_{k}(u-\varphi) \mathrm{d} x \leq \int_{\Omega} g T_{k}(u-\varphi) \mathrm{d} x
$$

for every $\varphi$ in $W_{0}^{1, p}(\Omega) \cap L^{\infty}(\Omega)$, and for every $k>0$.

We recall the following result (see [6], Th. 6.1, Th. 5.1 and Cor. 4.3).

Theorem 2.10. There exists a unique entropy solution of (2.12). Moreover this solution is also a solution of (2.12) in the sense of distributions. 


\subsection{Nonlinear problem}

The first main result (proved in Sect. 3) of the paper is the following:

Theorem 2.11. Let a satisfy the assumptions above, $A$ be an $N$-function satisfying the assumptions of Definition 2.1, with $q_{1}=p, q_{2}=N$, and let $\lambda$ be a bounded measure concentrated on a set $E$ of null $A$ capacity. Let $f_{n}$ be a sequence of functions converging to $\lambda$ in the sense of Definition 2.7. Let $g$ be a function in $L^{1}(\Omega)$, and let $g_{n}$ be a sequence of $L^{\infty}(\Omega)$ functions which converges to $g$ weakly in $L^{1}(\Omega)$. Let $\Phi \in C^{2}([0, \infty[)$ be an $N$-function such that

$$
\begin{gathered}
\Phi^{\prime}(t) \leq t \Phi^{\prime \prime}(t) \quad \forall t \geq 0 \\
\int^{+\infty} \frac{(\widetilde{A})^{\prime}(t) \Phi^{-1}\left(t^{p^{\prime}}\right)}{t^{p^{\prime}}} \mathrm{d} t<+\infty,
\end{gathered}
$$

and let $u_{n}$ be the solution in $W_{0}^{1, p}(\Omega)$ of the problem

$$
\left\{\begin{array}{cl}
-\operatorname{div}\left(a\left(x, \nabla u_{n}\right)\right)+\Phi^{\prime \prime}\left(\left|u_{n}\right|\right) u_{n}=f_{n}+g_{n} & \text { in } \Omega \\
u_{n}=0 & \text { on } \partial \Omega .
\end{array}\right.
$$

Then, as $n$ tends to infinity, $\left|\nabla u_{n}\right|^{p-1}$ converges strongly to $|\nabla u|^{p-1}$ in $L^{\Theta}(\Omega)$, for every $N$-function $\Theta \in \Delta_{2}$ such that

where $u$ is the unique entropy solution of

$$
\int^{+\infty} \frac{\Theta^{\prime}(t) \Phi^{-1}\left(t^{p^{\prime}}\right)}{t^{p^{\prime}}} \mathrm{d} t<+\infty
$$

$$
\left\{\begin{array}{cl}
-\operatorname{div}(a(x, \nabla u))+\Phi^{\prime \prime}(|u|) u=g & \text { in } \Omega \\
u=0 & \text { on } \partial \Omega .
\end{array}\right.
$$

Moreover,

$$
\lim _{n \rightarrow+\infty} \int_{\Omega} \Phi^{\prime \prime}\left(u_{n}\right) u_{n} \varphi \mathrm{d} x=\int_{\Omega} \Phi^{\prime \prime}(u) u \varphi \mathrm{d} x+\int_{\Omega} \varphi \mathrm{d} \lambda, \forall \varphi \in C_{\mathrm{c}}^{0}(\Omega)
$$

Remark 2.12. According to Theorem 2.10, Theorem 2.11 is also true with solutions in the distributional sense (but there is no uniqueness result).

Remark 2.13. Assumption (2.13) implies that the growth of the lower order term of the equation is linear or superlinear (observe that $\Phi^{\prime}(t) / t$ is non-decreasing because of (2.13)).

Remark 2.14. Let us now consider the Theorem 2.11 in the case $\Phi(t)=t^{q+1}$. The condition (2.14) can be reformulated as follows:

$$
\int^{+\infty} \frac{(\widetilde{A})^{\prime}(s)}{s^{\frac{p^{\prime} q}{q+1}}} \mathrm{~d} s<+\infty .
$$

Remark 2.15. Set $\Phi(t)=t^{q+1}$ and $A(t)=t^{r}$ (thus $\left.(\widetilde{A})^{\prime}(t)=c t^{1 /(r-1)}\right)$, then (2.14) (or (2.19)) becomes:

$$
\int^{+\infty} t^{\frac{1}{r-1}+\frac{p^{\prime}}{q+1}-p^{\prime}} \mathrm{d} t<+\infty
$$

This condition can be true only if $r>p$. In this case, this condition is equivalent to

$$
q>\frac{r(p-1)}{r-p}
$$


and the problem studied in Theorem 2.11 is

$$
\left\{\begin{array}{cl}
-\operatorname{div}(a(x, \nabla u))+|u|^{q-1} u=\mu & \text { in } \Omega, \\
u=0 & \text { on } \partial \Omega
\end{array}\right.
$$

i.e. the same condition found in [23], therefore our Theorem 2.11 is a generalization of Theorem 1.6 of [23].

Remark 2.16. Set $\Phi(t)=t^{q+1}$ and $A(t)=t^{r} \log ^{r-1+\varepsilon}(e+t)$ for some $\varepsilon>0$. There exist some constants $c_{1}, \ldots$, $c_{3}^{\prime}$, such that one has

$$
c_{1} t^{r-1} \log ^{r-1+\varepsilon}(e+t) \leq A^{\prime}(t) \leq c_{2} t^{r-1} \log ^{r-1+\varepsilon}(e+t), \quad \forall t>c_{3}
$$

and therefore (see Def. 2.4)

$$
c_{1}^{\prime} t^{\frac{1}{r-1}} \log ^{-\frac{r-1+\varepsilon}{r-1}}(e+t) \leq(\widetilde{A})^{\prime}(t) \leq c_{2}^{\prime} t^{\frac{1}{r-1}} \log ^{-\frac{r-1+\varepsilon}{r-1}}(e+t), \quad \forall t>c_{3}^{\prime}
$$

thus (2.14) (or (2.19)) becomes

$$
\int^{+\infty} t^{\frac{1}{r-1}+\frac{p^{\prime}}{q+1}-p^{\prime}} \log ^{-\frac{r-1+\varepsilon}{r-1}}(e+t) \mathrm{d} t<+\infty
$$

If $r>p$, this is equivalent to

$$
q \geq \frac{r(p-1)}{r-p}
$$

Remark 2.17. Let $p<r \leq N$, and $A(t)=t^{r}$. Then (2.15) has not solutions (in the sense of Th. 2.11) for any $\Phi$ such that

$$
\int^{+\infty} \frac{\Phi^{-1}\left(t^{p^{\prime}}\right)}{t^{p^{\prime}-r^{\prime}+1}} \mathrm{~d} t<+\infty
$$

We give here some examples of functions $\Phi$ for which (2.14) or (2.22) apply:

$$
\begin{aligned}
& \Phi_{1}(t)=t^{q+1} \quad \forall q>\frac{r(p-1)}{r-p} ; \\
& \Phi_{2}(t)=t^{\frac{r(p-1)}{r-p}+1}[\log (e+t)]^{k+1} \quad \forall k>\frac{r(p-1)}{r-p} ; \\
& \Phi_{3}(t)=t^{\frac{r(p-1)}{r-p}+1}[\log (e+t)]^{\frac{r(p-1)}{r-p}+1}[\log (e+\log (e+t))]^{k+1} \quad \forall k>\frac{r(p-1)}{r-p} ; \\
& \Phi_{4}(t)=e^{t}-t-1 ; \\
& \Phi_{5}(t)=e^{e^{t}-1}-t-1 .
\end{aligned}
$$

Remark 2.18. If $A(t)=t^{r}$ with $r \leq p$ then (2.14) has no solution in $\Phi$ (recall that $\Phi^{-1}$ is increasing). Moreover, in this case we have existence for (2.21), see Remark 1.10 of [23]. This motivates the bound $q_{1}=p$ in Theorem 2.11. Notice also that $q_{2}$ cannot be bigger than $N$ because there is not set of $r$-capacity null for $r>N$.

\subsection{Linear problem}

Let now study the linear case (where $p=2$ ) with $\Phi(t)=t^{q+1}$ :

$$
\left\{\begin{array}{cl}
-\Delta u+|u|^{q-1} u=\mu & \text { in } \Omega, \\
u=0 & \text { on } \partial \Omega .
\end{array}\right.
$$


If $A(t)=t^{r}$, according to Remark 2.15, equation (2.14) becomes $q>\frac{r}{r-2}\left(\right.$ or $\left.r>2 q^{\prime}\right)$. When $r=N$, one can see that Theorem 2.11 is thus weaker than the one of [9], where the condition is $q \geq \frac{N}{N-2}$. However if we set $A_{r}(t)=t^{r} \log ^{r-1+\varepsilon}(e+t)$, according to Remark 2.16, equation (2.14) becomes $q \geq \frac{r}{r-2}$. Therefore the capacities $\operatorname{cap}_{1, A_{r}}$ give us the possibility to allow also the case $q=\frac{r}{r-2}$ in Theorem 2.11:

Proposition 2.19. Let $\mu$ be a bounded measure concentrated on a set of null $\left(1, t^{r} \log ^{r-1+\varepsilon}(e+t)\right)$-capacity (for some $\varepsilon>0$ ) and absolutely continuous with respect to the $\left(1, t^{s}\right)$-capacity for all $s>r$. If $q \geq \frac{r}{r-2}$, then (2.23) has no solution in the sense of Theorem 2.11 and $\mu$ is not absolutely continuous with respect to the $\left(2, q^{\prime}\right)$-capacity. If $q<r /(r-2)$, then there exist solutions of (2.23) and $\mu$ is absolutely continuous with respect to the $\left(2, q^{\prime}\right)$-capacity.

Remark 2.20. Let us observe that this does not cover the case of [9]: the measure was concentrated on $\{0\}$, which is a set such that $\operatorname{cap}_{N}(\{0\})=\operatorname{cap}_{2, N / 2}(\{0\})=0, \operatorname{cap}_{1, t^{N} \log ^{N-1+\varepsilon}(e+t)}(\{0\})>0$ and $\operatorname{cap}_{s}(\{0\})>0$ for $s>N$. That is the Dirac mass is absolutely continuous with respect to the $\left(1, t^{N} \log ^{N-1+\varepsilon}(e+t)\right)$ and $(1, s)$-capacity for $s>N$ but not absolutely continuous with respect to the $(1, N)$ and $(2, N / 2)$-capacity.

Before proving the Proposition 2.19, we have to study the relations between the different capacities. This will be made by proving the following result, obtained by extending in the Orlicz setting the nonlinear potential techniques of [1] (see the proof in Sect. 4):

Theorem 2.21. Let $s>1,0<\beta s<N, \beta \in \mathbf{N}$. If

$$
\int_{0}^{1}(\widetilde{A})^{\prime}\left(t^{1-\beta s}\right) \mathrm{d} t<\infty
$$

then

$$
\operatorname{cap}_{1, A}(E)=0 \Rightarrow \operatorname{cap}_{\beta, s}(E)=0 .
$$

Remark 2.22. In order to prove Theorem 2.21, we will use a well-known result by Frostman (see (4.5) and (4.6)), which has been extended in Maz'ja and Havin [22]. We refer also to [20,21] for estimates close to ours, obtained by a completely different approach.

Setting $\beta=2$ and $s=q^{\prime}$ (with $2 q^{\prime}<N$ ) in Theorem 2.21, we get the following: if

$$
\int_{0}(\widetilde{A})^{\prime}\left(t^{1-2 q^{\prime}}\right) \mathrm{d} t<\infty
$$

then

$$
\operatorname{cap}_{1, A}(E)=0 \Rightarrow \operatorname{cap}_{2, q^{\prime}}(E)=0 .
$$


Therefore, as a consequence of the previous theorem, taking particular cases of the parameters involved, we get the following (already known) remark (see [23]):

Remark 2.23. Set $A(t)=t^{r}$. If $q>\frac{r}{r-2}$ (or equivalently $r>2 q^{\prime},(2.14,2.19)$ or $(2.25)$ ) in the Theorem 2.21, then we have

$$
\operatorname{cap}_{1, r}(E)=0 \Rightarrow \operatorname{cap}_{2, q^{\prime}}(E)=0 .
$$

Moreover if $\lambda$ is a measure concentrated on a set $E$ of null $(1, r)$-capacity then $\lambda$ is not absolutely continuous with respect to the $\left(2, q^{\prime}\right)$-capacity.

Let us go further studying the relation between these capacities:

Lemma 2.24. Let $A(t)=t^{r} \log ^{r-1+\varepsilon}(e+t)$ for some $\varepsilon>0$ and $q$ such that $2 q^{\prime}<N$ (i.e. $\left.q>\frac{N}{N-2}\right)$. If $q \geq \frac{r}{r-2}$ that is (2.14) or (2.25), one has according to Theorem 2.21,

$$
\operatorname{cap}_{1, t^{r} \log ^{r-1+\varepsilon}(e+t)}(E)=0 \Rightarrow \operatorname{cap}_{2, q^{\prime}}(E)=0 .
$$

If $q<\frac{r}{r-2}$ one has

$$
\operatorname{cap}_{2, q^{\prime}}(E)=0 \Rightarrow \operatorname{cap}_{1,2 q^{\prime}}(E)=0 \Rightarrow \operatorname{cap}_{1, t^{r} \log ^{r-1+\varepsilon}(e+t)}(E)=0 .
$$

Proof. If $q \geq \frac{r}{r-2}$, using Theorem 2.21 with $\beta=2$ and $s=q^{\prime}$, and $A(t)=t^{r} \log ^{r-1+\varepsilon}(e+t)$, one gets (2.26). If $q<\frac{r}{r-2}$, then $r<2 q^{\prime}$, from which $A(t) \leq t^{2 q^{\prime}}$ near infinity. Thus

$$
A^{-1}\left(\operatorname{cap}_{1, t^{r} \log ^{r-1+\varepsilon}(e+t)}(E)\right) \leq c\left[\operatorname{cap}_{1,2 q^{\prime}}(E)\right]^{1 / 2 q^{\prime}},
$$

and according to Adams-Hedberg [1] (Th. 5.5.1, p. 148), one has

$$
\operatorname{cap}_{1, t^{r} \log ^{r-1+\varepsilon}(e+t)}(E) \leq c A\left(\left[\operatorname{cap}_{1,2 q^{\prime}}(E)\right]^{1 / 2 q^{\prime}}\right) \leq c A\left(\left[\operatorname{cap}_{2, q^{\prime}}(E)\right]^{1 / 2 q^{\prime}}\right) .
$$

We are now able to prove the Proposition 2.19.

Proof of Proposition 2.19. In the case $q \geq \frac{r}{r-2}$, equation (2.14) is true, and $\mu$ is concentrated on a set of null $\left(1, t^{r} \log ^{r-1+\varepsilon}(e+t)\right)$-capacity, then (2.23) has no solution in the sense of Theorem 2.11 (using it with $\left.A(t)=t^{r} \log ^{r-1+\varepsilon}(e+t)\right)$. Moreover using the first part of Lemma 2.24, one has that $\mu$ is concentrated on a set of null $\left(2, q^{\prime}\right)$-capacity and thus $\mu$ is not absolutely continuous with respect to this capacity.

In the case $q<r /(r-2), 2 q^{\prime}>r$ then $\mu$ is absolutely continuous with respect to the $\left(1,2 q^{\prime}\right)$-capacity (since $\left.2 q^{\prime}>r\right)$ and thus also to the $\left(2, q^{\prime}\right)$-capacity (according to Lem. 2.24), then there exist solutions of (2.23) (see Gallouët and Morel [14]).

\section{Proof of the NONEXistence RESUlT}

Before giving the proof of Theorem 2.11, we need to construct, as in [11], a sequence of suitable cut-off functions, built after $\lambda$ and $E$ (the proof of [23] works also for Sobolev-Orlicz spaces).

Lemma 3.1. Let $\lambda=\lambda^{+}-\lambda^{-}$be a Radon measure concentrated on a set $E$ of zero $r$-capacity, with $1<r \leq N$. Then for every $\delta>0$ there exist two $C_{\mathrm{c}}^{\infty}(\Omega)$ function $\psi_{\delta}^{+}$and $\psi_{\delta}^{-}$such that

$$
\begin{gathered}
0 \leq \psi_{\delta}^{+} \leq 1,0 \leq \psi_{\delta}^{-} \leq 1, \quad\left\|\nabla \psi_{\delta}^{+}\right\|_{A} \leq \delta,\left\|\nabla \psi_{\delta}^{-}\right\|_{A} \leq \delta, \\
0 \leq \int_{\Omega}\left(1-\psi_{\delta}^{+}\right) \mathrm{d} \lambda^{+} \leq \delta, \quad 0 \leq \int_{\Omega}\left(1-\psi_{\delta}^{-}\right) \mathrm{d} \lambda^{-} \leq \delta \\
0 \leq \int_{\Omega} \psi_{\delta}^{-} \mathrm{d} \lambda^{+} \leq \delta, \quad 0 \leq \int_{\Omega} \psi_{\delta}^{+} \mathrm{d} \lambda^{-} \leq \delta
\end{gathered}
$$


Lemma 3.2. Let $\rho>0$, and let $\left\{v_{n}\right\}$ be a sequence of functions bounded in $M^{\Phi^{\prime}}(\Omega)$. Suppose that, for every $k>0$, we have

$$
\int_{\Omega}\left|\nabla T_{k}\left(v_{n}\right)\right|^{p} \mathrm{~d} x \leq c k
$$

for some positive constant c. Then $\left\{\left|\nabla v_{n}\right|^{p-1}\right\}$ is bounded in $M^{\Psi}(\Omega)$, with

$$
\Psi(s)=\frac{s^{p^{\prime}}}{\Phi^{-1}\left(s^{p^{\prime}}\right)} .
$$

Proof. We follow the lines of the proof of [6] (Lem. 4.2). Let $\sigma$ be a fixed positive real number. We have, for every $k>0$,

$$
\begin{aligned}
\text { meas }\left\{\left|\nabla v_{n}\right|>\sigma\right\} & =\text { meas }\left\{\begin{array}{c}
\left|\nabla v_{n}\right|>\sigma \\
\left|v_{n}\right| \leq k
\end{array}\right\}+\text { meas }\left\{\begin{array}{c}
\left|\nabla v_{n}\right|>\sigma \\
\left|v_{n}\right|>k
\end{array}\right\} \\
& \leq \text { meas }\left\{\begin{array}{c}
\left|\nabla v_{n}\right|>\sigma \\
\left|v_{n}\right| \leq k
\end{array}\right\}+\text { meas }\left\{\left|v_{n}\right|>k\right\}
\end{aligned}
$$

Moreover,

$$
\operatorname{meas}\left\{\begin{array}{c}
\left|\nabla v_{n}\right|>\sigma \\
\left|v_{n}\right| \leq k
\end{array}\right\} \leq \frac{1}{\sigma^{p}} \int_{\Omega}\left|\nabla T_{k}\left(v_{n}\right)\right|^{p} \mathrm{~d} x \leq c \frac{k}{\sigma^{p}} .
$$

Since by the assumptions on $v_{n}$ there exists a positive constant $c$ such that

$$
\operatorname{meas}\left\{\left|v_{n}\right|>k\right\} \leq \frac{c}{\Phi^{\prime}(k)}
$$

equation (3.4) then implies

$$
\text { meas }\left\{\left|\nabla v_{n}\right|>\sigma\right\} \leq c \frac{k}{\sigma^{p}}+\frac{c}{\Phi^{\prime}(k)},
$$

and this latter inequality holds for every $k>0$. Minimizing on $k$, we get $c k \Phi^{\prime}(k)=\sigma^{p}$ (recall that $k \Phi^{\prime}(k)$ $\geq \Phi(k)$ and $\Phi^{-1}(c k) \leq c \Phi^{-1}(k)$, for all $c>1$, thanks to the convexity of $\left.\Phi\right)$

$$
\operatorname{meas}\left\{\left|\nabla v_{n}\right|>\sigma\right\} \leq \frac{c \Phi^{-1}\left(\sigma^{p}\right)}{\sigma^{p}}
$$

thus

which is the desired result.

$$
\operatorname{meas}\left\{\left|\nabla v_{n}\right|^{p-1}>\sigma\right\} \leq \frac{c \Phi^{-1}\left(\sigma^{p^{\prime}}\right)}{\sigma^{p^{\prime}}},
$$

Lemma 3.3. Let $\Psi$ and $\Theta$ be $N$-functions. If moreover

$$
\int^{+\infty} \frac{\Theta^{\prime}(t)}{\Psi(t)} \mathrm{d} t<+\infty
$$

then one has

$$
M^{\Psi}(\Omega) \subset L^{\Theta}(\Omega)
$$

and for any $s>0$ the following inequality hold:

$$
\int_{\Omega} \Theta(|v|) \mathrm{d} x \leq \operatorname{meas}(\Omega) \Theta(s)+\left(\sup _{t>0} \Psi(t) \text { meas }\{|v|>t\}\right) \int_{s}^{+\infty} \frac{\Theta^{\prime}(t)}{\Psi(t)} \mathrm{d} t .
$$


Proof. Let $v$ be a function in $M^{\Psi}(\Omega)$. One has, for all $s>0$,

$$
\begin{aligned}
\int_{\Omega} \Theta(|v|) \mathrm{d} x & =\int_{0}^{+\infty} \Theta^{\prime}(t) \text { meas }\{|v|>t\} \mathrm{d} t \\
& \leq \operatorname{meas}(\Omega) \Theta(s)+\int_{s}^{+\infty} \frac{\Theta^{\prime}(t)}{\Psi(t)} \Psi(t) \text { meas }\{|v|>t\} \mathrm{d} t
\end{aligned}
$$

from which the assertion follows.

Lemma 3.4. Let $\left\{v_{n}\right\}$ be a sequence of $W_{0}^{1, p}(\Omega)$ functions such that

$$
\int_{\Omega}\left|\nabla T_{k}\left(v_{n}\right)\right|^{p} \mathrm{~d} x \leq c k,
$$

for some positive constant $c$. Then there exists a subsequence, still denoted by $v_{n}$, and a measurable function $v$, such that $v_{n}$ converges to $v$ almost everywhere in $\Omega$.

Proof. See [6], proof of Theorem 6.1, Step 2.

Proof of Theorem 2.11. We will follow [23] which has used some of the ideas contained in [11] when dealing with nonlinear elliptic equations with measure data.

Then, since the operator is monotone, there exists a unique solution $u$ in $W_{0}^{1, p}(\Omega)$ of the following nonlinear elliptic problem (this result is well known and is a consequence of [18]; it is, for example, proved in Th. 2.10)

$$
\left\{\begin{array}{cl}
-\operatorname{div}(a(x, \nabla u))+\Phi^{\prime \prime}(|u|) u=f & \text { in } \Omega, \\
u=0 & \text { on } \partial \Omega,
\end{array}\right.
$$

in the sense that

$$
\int_{\Omega} a(x, \nabla u) \cdot \nabla \varphi \mathrm{d} x+\int_{\Omega} \Phi^{\prime \prime}(|u|) u \varphi \mathrm{d} x=\int_{\Omega} f \varphi \mathrm{d} x,
$$

for every $\varphi$ in $W_{0}^{1, p}(\Omega) \cap L^{\infty}(\Omega)$ and for $\varphi=u$, so that $\Phi(|u|)$ (and $\Phi^{\prime \prime}(|u|) u^{2}$ ) belongs to $L^{1}(\Omega)$.

We define $\omega(n, m, \delta)$ any quantity (depending on $n, m$ and $\delta$ ) such that

$$
\lim _{\delta \rightarrow 0^{+}} \lim _{m \rightarrow+\infty} \lim _{n \rightarrow+\infty}|\omega(n, m, \delta)|=0 .
$$

Similarly, if the quantity we are considering does not depend one or more of the three parameters $n, m$ and $\delta$, we will omit the dependence from it in $\omega$. For example, $\omega(n, \delta)$ is any quantity such that

$$
\lim _{\delta \rightarrow 0^{+}} \lim _{n \rightarrow+\infty}|\omega(n, \delta)|=0 .
$$

Step 1: A priori estimates.

Since $T_{k}\left(u_{n}\right)$ is in $W_{0}^{1, p}(\Omega) \cap L^{\infty}(\Omega)$, we can choose it as test function in the weak formulation of (2.15). We get, using $(2.7,2.11)$, and the boundedness of $\left\{g_{n}\right\}$ in $L^{1}(\Omega)$,

$$
\alpha \int_{\Omega}\left|\nabla T_{k}\left(u_{n}\right)\right|^{p} \mathrm{~d} x+\int_{\Omega} \Phi^{\prime \prime}\left(\left|u_{n}\right|\right)\left|u_{n}\right|\left|T_{k}\left(u_{n}\right)\right| \mathrm{d} x \leq c k,
$$

for some positive constant $c$. Dropping the first, nonnegative term of the left hand side of the preceding inequality, we have

$$
k \int_{\left\{\left|u_{n}\right| \geq k\right\}} \Phi^{\prime \prime}\left(\left|u_{n}\right|\right)\left|u_{n}\right| \mathrm{d} x \leq \int_{\Omega} \Phi^{\prime \prime}\left(\left|u_{n}\right|\right)\left|u_{n}\right|\left|T_{k}\left(u_{n}\right)\right| \mathrm{d} x \leq c k,
$$


so that

By (2.13) this implies

$$
\int_{\left\{\left|u_{n}\right| \geq k\right\}} \Phi^{\prime \prime}\left(\left|u_{n}\right|\right)\left|u_{n}\right| \mathrm{d} x \leq c
$$

and so $\left\{u_{n}\right\}$ is bounded in $M^{\Phi^{\prime}}(\Omega)$. Furthermore,

$$
\Phi^{\prime}(k) \text { meas }\left\{\left|u_{n}\right| \geq k\right\} \leq k \Phi^{\prime \prime}(k) \text { meas }\left\{\left|u_{n}\right| \geq k\right\} \leq c,
$$

$$
\int_{\left\{\left|u_{n}\right|<k\right\}} \Phi^{\prime \prime}\left(\left|u_{n}\right|\right)\left|u_{n}\right| \mathrm{d} x \leq k \Phi^{\prime \prime}(k) \text { meas }(\Omega),
$$

and so, using (3.8),

$$
\Phi^{\prime \prime}\left(\left|u_{n}\right|\right) u_{n} \text { is bounded in } L^{1}(\Omega) .
$$

The boundedness of $u_{n}$ in $M^{\Phi^{\prime}}(\Omega)$, and Lemma 3.2, which can be applied since (3.7) also implies that

$$
\int_{\Omega}\left|\nabla T_{k}\left(u_{n}\right)\right|^{p} \mathrm{~d} x \leq c k
$$

yields

$$
\left\{\left|\nabla u_{n}\right|^{p-1}\right\} \text { is bounded in } M^{\Psi}(\Omega), \text { with } \Psi(s)=\frac{s^{p^{\prime}}}{\Phi^{-1}\left(s^{p^{\prime}}\right)} .
$$

Now let $\Theta_{1} \in \Delta_{2}$ be an $N$-function verifying the assumption (2.16), and let $\Theta \in \Delta_{2}$ be any $N$-function "well dominated" by $\Theta_{1}$. We formalize this domination writing

$$
\Theta_{1}(t)=\varphi(\Theta(t)) \quad \forall t \geq 0
$$

for some $\varphi$ increasing, continuous, such that $\lim _{t \rightarrow \infty} \varphi(t) / t=+\infty$. By (3.11) and Lemma 3.3 the set $\left\{\varphi\left(\Theta\left(\left|\nabla u_{n}\right|^{p-1}\right)\right)\right\}$ is bounded in $L^{1}(\Omega)$, therefore the sequence

$$
\Theta\left(\left|\nabla u_{n}\right|^{p-1}\right) \text { is equiintegrable. }
$$

On the other hand, using again (3.10), by Lemma 3.4, and up to some subsequence still denoted by $u_{n}, u_{n}$ converges almost everywhere to a measurable function $u$, and so $T_{k}\left(u_{n}\right)$ converges almost everywhere to $T_{k}(u)$. Using (3.9) and Fatou lemma, one has $\Phi^{\prime \prime}(|u|) u \in L^{1}(\Omega)$.

Moreover, equation (3.10) implies that $\left\{T_{k}\left(u_{n}\right)\right\}$ is bounded in $W_{0}^{1, p}(\Omega)$, so that, by the weak lower semicontinuity of the norm, $T_{k}(u)$ belongs to $W_{0}^{1, p}(\Omega)$ for every $k>0$, and thus $u$ has a gradient $\nabla u$ in the sense of Definition 2.8.

As for the gradients of $u_{n}$, we remark that $u_{n}$ is the solution of the equation $-\operatorname{div}\left(a\left(x, \nabla u_{n}\right)\right)=f_{n}^{\oplus}-f_{n}^{\ominus}$ $+g_{n}-\Phi^{\prime \prime}\left(\left|u_{n}\right|\right) u_{n}$, and that the right hand side is bounded in $L^{1}(\Omega)$ by (2.11) and (3.9). By a result in [8], this implies that, up to subsequences,

$$
\nabla u_{n} \text { converges almost everywhere to } \nabla u \text {. }
$$

From now on, we will suppose to have already extracted from $u_{n}$ a subsequence (which we still denote by $u_{n}$ ), with the properties we have proved before. By (3.13) we have also

$$
\Theta\left(\left|\nabla u_{n}\right|^{p-1}\right) \text { converges almost everywhere to } \Theta\left(|\nabla u|^{p-1}\right) \text {. }
$$

By (3.12) and (3.14), we can apply Vitali's theorem, and we get $\left|\nabla u_{n}\right|^{p-1} \in L^{\Theta}(\Omega)$ and

$$
\int_{\Omega} \Theta\left(\left|\nabla u_{n}\right|^{p-1}\right) \mathrm{d} x \rightarrow \int_{\Omega} \Theta\left(|\nabla u|^{p-1}\right) \mathrm{d} x
$$


By (3.13) and (3.15), applying the Fatou lemma to the sequence of nonnegative functions $c_{\Theta}\left(\Theta\left(|\nabla u|^{p-1}\right)\right.$ $\left.+\Theta\left(\left|\nabla u_{n}\right|^{p-1}\right)\right)-\Theta\left(\left.|| \nabla u\right|^{p-1}-\left|\nabla u_{n}\right|^{p-1} \mid\right)$, where $c_{\Theta}$ is the constant appearing in the $\Delta_{2}$ condition for $\Theta$, we get

$$
\int_{\Omega} \Theta\left(\left.|| \nabla u\right|^{p-1}-\left|\nabla u_{n}\right|^{p-1} \mid\right) \mathrm{d} x \rightarrow 0
$$

from which, since $\Theta \in \Delta_{2}$, we get (see e.g. Th. 1.3, p. 8 of [19])

$$
\left|\nabla u_{n}\right|^{p-1} \rightarrow|\nabla u|^{p-1} \quad \text { strongly in } L^{\Theta}(\Omega) .
$$

Notice that we obtained (3.17) for all $\Theta \in \Delta_{2}$ well dominated by some $\Theta_{1}$ such that (2.16) holds. Such functions $\Theta \in \Delta_{2}$ verify condition (2.16), and, on the other hand, arguing as in [17] (see Chap. II, Sect. 8, No. 1, p. 60), it is easy to show that any $\Delta_{2} N$-function satisfying the condition (2.16) is well dominated by an $N$-function of the same type. The conclusion is that we have (3.17) for all $\Theta$ verifying (2.16).

Observe that, by the assumption (2.8) on $a$, the argument above shows also that

$$
a\left(x, \nabla u_{n}\right) \rightarrow a(x, \nabla u) \quad \text { strongly in }\left(L^{\Theta}(\Omega)\right)^{N},
$$

for every function $\Theta \in \Delta_{2}$ such that $\int^{+\infty} \frac{\Theta^{\prime}(t)}{\Psi(t)} \mathrm{d} t<\infty$. In particular, one can choose $\Theta=\widetilde{A}$ thanks to (2.14). Thus the last convergence is also in $L^{1}(\Omega)$.

Step 2: Energy estimates.

Let $\Psi_{\delta}=\psi_{\delta}^{+}+\psi_{\delta}^{-}$, where $\psi_{\delta}^{+}$and $\psi_{\delta}^{-}$are as in Lemma 3.1. Then

$$
\int_{\left\{u_{n}>2 m\right\}} \Phi^{\prime \prime}\left(u_{n}\right) u_{n}\left(1-\Psi_{\delta}\right) \mathrm{d} x=\omega(n, m, \delta),
$$

and

$$
\int_{\left\{u_{n}<-2 m\right\}} \Phi^{\prime \prime}\left(\left|u_{n}\right|\right)\left|u_{n}\right|\left(1-\Psi_{\delta}\right) \mathrm{d} x=\omega(n, m, \delta) .
$$

We will only prove (3.19), since the proof of (3.20) is identical. We choose $\beta_{m}\left(u_{n}\right)\left(1-\Psi_{\delta}\right)$ as test function in the weak formulation of $(2.15)$, where $\beta_{m}(s)$ is defined as

$$
\beta_{m}(s)=\left\{\begin{array}{cl}
0 & \text { if } s \leq m \\
\frac{s}{m}-1 & \text { if } m<s \leq 2 m \\
1 & \text { if } s>2 m
\end{array}\right.
$$


We obtain, using the fact that the derivative of $\beta_{m}(s)$ is different from zero only where $m<s<2 m$,

$$
\begin{aligned}
& \frac{1}{m} \int_{\left\{m<u_{n}<2 m\right\}} a\left(x, \nabla u_{n}\right) \cdot \nabla u_{n}\left(1-\Psi_{\delta}\right) \mathrm{d} x \\
& \quad-\int_{\Omega} a\left(x, \nabla u_{n}\right) \cdot \nabla \Psi_{\delta} \beta_{m}\left(u_{n}\right) \mathrm{d} x \\
& \quad+\int_{\Omega} \Phi^{\prime \prime}\left(\left|u_{n}\right|\right) u_{n} \beta_{m}\left(u_{n}\right)\left(1-\Psi_{\delta}\right) \mathrm{d} x \\
& =\int_{\Omega} f_{n}^{\oplus} \beta_{m}\left(u_{n}\right)\left(1-\Psi_{\delta}\right) \mathrm{d} x \\
& \quad-\int_{\Omega} f_{n}^{\ominus} \beta_{m}\left(u_{n}\right)\left(1-\Psi_{\delta}\right) \mathrm{d} x \\
& \quad+\int_{\Omega} g_{n} \beta_{m}\left(u_{n}\right)\left(1-\Psi_{\delta}\right) \mathrm{d} x .
\end{aligned}
$$

We have, by (3.18), by Egorov theorem, and since $\beta_{m}\left(u_{n}\right)$ converges to $\beta_{m}(u)$ almost everywhere in $\Omega$ and in the weak* topology of $L^{\infty}(\Omega)$,

$$
-(B)=\int_{\Omega} a(x, \nabla u) \cdot \nabla \Psi_{\delta} \beta_{m}(u) \mathrm{d} x+\omega(n)=\omega(n, m),
$$

and the last passage is due to the fact that $\beta_{m}(u)$ converges to zero in the weak* topology of $L^{\infty}(\Omega)$ as $m$ tends to infinity. For the same reason, we have

$$
(F)=\omega(n, m) .
$$

Finally, by (3.2) and (3.3),

$$
\begin{aligned}
(D) & \leq \int_{\Omega} f_{n}^{\oplus}\left(1-\Psi_{\delta}\right) \mathrm{d} x=\int_{\Omega} f_{n}^{\oplus}\left(1-\psi_{\delta}^{+}\right) \mathrm{d} x+\int_{\Omega} f_{n}^{\oplus} \psi_{\delta}^{-} \mathrm{d} x \\
& =\int_{\Omega}\left(1-\psi_{\delta}^{+}\right) \mathrm{d} \lambda^{+}+\int_{\Omega} \psi_{\delta}^{-} \mathrm{d} \lambda^{+}+\omega(n) \\
& =\omega(n, \delta) .
\end{aligned}
$$

Since (A) and $-(\mathrm{E})$ are nonnegative, and since

$$
(C) \geq \int_{\left\{u_{n}>2 m\right\}} \Phi^{\prime \prime}\left(u_{n}\right) u_{n}\left(1-\Psi_{\delta}\right) \mathrm{d} x,
$$

we get (3.19).

Step 3: Passing to the limit.

We are now ready to conclude the proof of Theorem 2.11, showing that $u$ is the entropy solution of (2.17) with datum $g$. 
Let $\varphi$ be a function in $W_{0}^{1, p}(\Omega) \cap L^{\infty}(\Omega)$, let $M=\|\varphi\|_{L^{\infty}(\Omega)}$, let $k>0$, and choose $T_{k}\left(u_{n}-\varphi\right)\left(1-\Psi_{\delta}\right)$ as test function in the weak formulation of (2.15). We get

$$
\begin{aligned}
& \int_{\Omega} a\left(x, \nabla T_{k}\left(u_{n}\right)\right) \cdot \nabla T_{k}\left(u_{n}-\varphi\right)\left(1-\Psi_{\delta}\right) \mathrm{d} x \\
& \quad-\int_{\Omega} a\left(x, \nabla u_{n}\right) \cdot \nabla \Psi_{\delta} T_{k}\left(u_{n}-\varphi\right) \mathrm{d} x \\
& \quad+\int_{\Omega} \Phi^{\prime \prime}\left(\left|u_{n}\right|\right) u_{n} T_{k}\left(u_{n}-\varphi\right)\left(1-\Psi_{\delta}\right) \mathrm{d} x \\
& =\int_{\Omega} f_{n}^{\oplus} T_{k}\left(u_{n}-\varphi\right)\left(1-\Psi_{\delta}\right) \mathrm{d} x \\
& \quad-\int_{\Omega} f_{n}^{\ominus} T_{k}\left(u_{n}-\varphi\right)\left(1-\Psi_{\delta}\right) \mathrm{d} x \\
& \quad+\int_{\Omega} g_{n} T_{k}\left(u_{n}-\varphi\right)\left(1-\Psi_{\delta}\right) \mathrm{d} x .
\end{aligned}
$$

Using $(3.18,2.14)$, Lemma 3.3 , one has the convergence of $a\left(x, \nabla u_{n}\right)$ to $a(x, \nabla u)$ in $L^{\widetilde{A}}(\Omega)$. Thus using (3.1), we get

$$
-(B)=\int_{\Omega} a(x, \nabla u) \cdot \nabla \Psi_{\delta} T_{k}(u-\varphi) \mathrm{d} x+\omega(n)=\omega(n, \delta) .
$$

Using (3.2) and (3.3), we obtain

$$
|(D)|+|(E)| \leq k \int_{\Omega}\left(f_{n}^{\oplus}+f_{n}^{\ominus}\right)\left(1-\Psi_{\delta}\right) \mathrm{d} x=\omega(n, \delta) .
$$

It is then easy to see that

$$
(F)=\int_{\Omega} g T_{k}(u-\varphi) \mathrm{d} x+\omega(n, \delta)
$$

so that we only have to deal with (A) and (B). Let $m>k+M$ be fixed. We then have

$$
\begin{aligned}
(\mathrm{C})= & \int_{\left\{-2 m \leq u_{n} \leq 2 m\right\}} \Phi^{\prime \prime}\left(\left|u_{n}\right|\right) u_{n} T_{k}\left(u_{n}-\varphi\right)\left(1-\Psi_{\delta}\right) \mathrm{d} x \\
& +\int_{\left\{u_{n}>2 m\right\}} \Phi^{\prime \prime}\left(u_{n}\right) u_{n} k\left(1-\Psi_{\delta}\right) \mathrm{d} x \\
& +\int_{\left\{u_{n}<-2 m\right\}} \Phi^{\prime \prime}\left(\left|u_{n}\right|\right)\left|u_{n}\right| k\left(1-\Psi_{\delta}\right) \mathrm{d} x .
\end{aligned}
$$

It is easily seen that (recall that $\Phi^{\prime \prime}(|u|) u \in L^{1}(\Omega)$ )

$$
\begin{aligned}
(G) & =\int_{\{-2 m \leq u \leq 2 m\}} \Phi^{\prime \prime}(|u|) u T_{k}(u-\varphi)\left(1-\Psi_{\delta}\right) \mathrm{d} x+\omega(n) \\
& =\int_{\Omega} \Phi^{\prime \prime}(|u|) u T_{k}(u-\varphi)\left(1-\Psi_{\delta}\right) \mathrm{d} x+\omega(n, m) \\
& =\int_{\Omega} \Phi^{\prime \prime}(|u|) u T_{k}(u-\varphi) \mathrm{d} x+\omega(n, m, \delta) .
\end{aligned}
$$

We then have, by (3.19),

$$
(H)=k \int_{\left\{u_{n}>2 m\right\}} \Phi^{\prime \prime}\left(u_{n}\right) u_{n}\left(1-\Psi_{\delta}\right) \mathrm{d} x=\omega(n, m, \delta),
$$


and, by (3.20),

so that

$$
(I)=k \int_{\left\{u_{n}<-2 m\right\}} \Phi^{\prime \prime}\left(\left|u_{n}\right|\right)\left|u_{n}\right|\left(1-\Psi_{\delta}\right) \mathrm{d} x=\omega(n, m, \delta),
$$

Finally, we have

$$
(C)=\int_{\Omega} \Phi^{\prime \prime}(|u|) u T_{k}(u-\varphi) \mathrm{d} x+\omega(n, \delta) .
$$

$$
\begin{aligned}
(A)= & \int_{\Omega}\left[a\left(x, \nabla u_{n}\right)-a(x, \nabla \varphi)\right] \cdot \nabla T_{k}\left(u_{n}-\varphi\right)\left(1-\Psi_{\delta}\right) \mathrm{d} x \\
& +\int_{\Omega} a(x, \nabla \varphi) \cdot \nabla T_{k}\left(u_{n}-\varphi\right)\left(1-\Psi_{\delta}\right) \mathrm{d} x
\end{aligned}
$$

Since the integrand function in $(\mathrm{J})$ is nonnegative, and converges almost everywhere in $\Omega$ to $[a(x, \nabla u)-a(x, \nabla \varphi)]$. $\nabla T_{k}(u-\varphi)$, as $n$ tends to infinity and then $\delta$ tends to zero, Fatou lemma implies

$$
\int_{\Omega}[a(x, \nabla u)-a(x, \nabla \varphi)] \cdot \nabla T_{k}(u-\varphi) \mathrm{d} x \leq \liminf _{\delta \rightarrow 0^{+}} \liminf _{n \rightarrow+\infty}(J) .
$$

Moreover, since $a(x, \nabla \varphi)$ belongs to $\left(L^{p^{\prime}}(\Omega)\right)^{N}$, we have

$$
(K)=\int_{\Omega} a(x, \nabla \varphi) \cdot \nabla T_{k}(u-\varphi) \mathrm{d} x+\omega(n, \delta),
$$

so that, putting together the results for $(\mathrm{J})$ and $(\mathrm{K})$, we have

$$
\int_{\Omega} a(x, \nabla u) \cdot \nabla T_{k}(u-\varphi) \mathrm{d} x \leq \liminf _{\delta \rightarrow 0^{+}} \liminf _{n \rightarrow+\infty}(A) .
$$

Summing up the results we have obtained so far, we have

$$
\int_{\Omega} a(x, \nabla u) \cdot \nabla T_{k}(u-\varphi) \mathrm{d} x+\int_{\Omega} \Phi^{\prime \prime}(|u|) u T_{k}(u-\varphi) \mathrm{d} x \leq \int_{\Omega} g T_{k}(u-\varphi) \mathrm{d} x,
$$

and so $u$ is the entropy solution of (2.17). Observe that, thanks to the uniqueness of entropy solution, the solution $u$ does not depend on the subsequences we have extracted, then the whole sequence $u_{n}$ converges to $u$.

To conclude the proof of the theorem, it only remains to prove (2.18). In order to do this, we choose a test function $\varphi \in C_{\mathrm{c}}^{\infty}(\Omega)$ in the weak formulation of (2.15). We get

$$
\int_{\Omega} a\left(x, \nabla u_{n}\right) \cdot \nabla \varphi \mathrm{d} x+\int_{\Omega} \Phi^{\prime \prime}\left(\left|u_{n}\right|\right) u_{n} \varphi \mathrm{d} x=\int_{\Omega}\left(f_{n}+g_{n}\right) \varphi \mathrm{d} x .
$$

Thanks to (3.18), and to the assumptions on $f_{n}$ and $g_{n}$, we have

$$
\int_{\Omega} \Phi^{\prime \prime}\left(\left|u_{n}\right|\right) u_{n} \varphi \mathrm{d} x=-\int_{\Omega} a(x, \nabla u) \cdot \nabla \varphi \mathrm{d} x+\int_{\Omega} g \varphi \mathrm{d} x+\int_{\Omega} \varphi \mathrm{d} \lambda+\omega(n) .
$$

Since the entropy solution of (2.17) is also a distributional solution of the same problem, we have for the same $\varphi$,

$$
\int_{\Omega} a(x, \nabla u) \cdot \nabla \varphi \mathrm{d} x+\int_{\Omega} \Phi^{\prime \prime}(|u|) u \varphi \mathrm{d} x=\int_{\Omega} g \varphi \mathrm{d} x,
$$

and so we have proved that (2.18) holds for every $\varphi$ in $C_{\mathrm{c}}^{\infty}(\Omega)$. Since $\Phi^{\prime \prime}\left(\left|u_{n}\right|\right) u_{n}$ is bounded in $L^{1}(\Omega)$, equation $(2.18)$ can then be extended by density to the functions in $C_{\mathrm{c}}^{0}(\Omega)$. 


\section{Proof of the COMPARISON RESUlt}

The proof of Theorem 2.21 is quite long, thus we will need first several intermediate results.

We give now some basic definitions, and fix some notation, borrowed mainly from the book by Adams and Hedberg [1]. The Fourier transform of a function $f$ will be denoted $\mathcal{F} f(\xi)$. If $f \in L^{1}\left(\mathbf{R}^{N}\right), \mathcal{F} f(\xi)=$ $\int_{\mathbf{R}^{N}} f(x) \mathrm{e}^{-i x \xi} \mathrm{d} x$. The Bessel kernel is defined by

$$
G_{\alpha}=\mathcal{F}^{-1}\left(\left(1+|\xi|^{2}\right)^{\frac{\alpha}{2}}\right) \quad(\alpha \in \mathbf{R})
$$

It can be shown that the following integral formula holds:

$$
G_{\alpha}(x)=\frac{1}{(4 \pi)^{\alpha / 2} \Gamma(\alpha / 2)} \int_{0}^{\infty} t^{(\alpha-N) / 2} \mathrm{e}^{-\pi|x|^{2} / t-t /(4 \pi)} \frac{\mathrm{d} t}{t} \quad(\alpha>0) .
$$

Moreover, $G_{\alpha}$ is positive and integrable over $\mathbf{R}^{N}$. The Hardy-Littlewood fractional maximal function of a measure $\mu$ for $0 \leq \alpha<N, \delta>0$, is defined by

$$
M_{\alpha, \delta} \mu(x)=\sup _{0<r \leq \delta} \frac{\mu(B(x, r))}{|B(x, r)|^{(N-\alpha) / N}} .
$$

In the sequel we will use the following inequality, trivial in the context of Lebesgue spaces (this inequality can be related to the inequality for Jensen means proved in [13]):

Lemma 4.1. The following inequality holds:

$$
A\left(\|f\|_{A}\right) \leq \Psi_{A}\left(\int_{\Omega} A(|f|) \mathrm{d} x\right)
$$

where

Moreover, the following bounds for $\Psi_{A}$ hold:

$$
\Psi_{A}(s)=\sup _{t>0} A\left(\frac{t}{A^{-1}\left(\frac{A(t)}{s}\right)}\right) .
$$

$$
c_{1} \min \left(A\left(s^{1 / q_{1}}\right), A\left(s^{1 / q_{2}}\right)\right) \leq \Psi_{A}(s) \leq c_{2} \max \left(A\left(s^{1 / q_{1}}\right), A\left(s^{1 / q_{2}}\right)\right) .
$$

Proof. By definition of $\Psi_{A}$ we have

$$
A\left(\frac{t}{A^{-1}\left(\frac{A(t)}{s}\right)}\right) \leq \Psi_{A}(s) \quad \forall s, t>0
$$

or, equivalently,

$$
\begin{array}{ll}
\frac{t}{A^{-1}\left(\frac{A(t)}{s}\right)} \leq A^{-1}\left(\Psi_{A}(s)\right) & \forall s, t>0 \\
\frac{t}{A^{-1}\left(\Psi_{A}(s)\right)} \leq A^{-1}\left(\frac{A(t)}{s}\right) & \forall s, t>0 \\
A\left(\frac{t}{A^{-1}\left(\Psi_{A}(s)\right)}\right) \leq \frac{A(t)}{s} & \forall s, t>0
\end{array}
$$


and therefore, replacing $t$ by $|f(x)|$ and $s$ by $\int_{\Omega} A(|f(x)|) \mathrm{d} x$ and integrating over $\Omega$

$$
\int_{\Omega} A\left(\frac{|f(x)|}{A^{-1}\left(\Psi_{A}\left(\int_{\Omega} A(|f(x)|) \mathrm{d} x\right)\right)}\right) \mathrm{d} x \leq 1 .
$$

By definition of Orlicz norm we deduce

$$
\|f\|_{A} \leq A^{-1}\left(\Psi_{A}\left(\int_{\Omega} A(|f(x)|) \mathrm{d} x\right)\right)
$$

from which the first part of the assertion follows. The two bounds for $\Psi_{A}$ can be proved in the same way, we will show only the upper one. By (2.2) we have

$$
\sigma t \leq c_{2} A^{-1}\left(\max \left(\sigma^{q_{1}}, \sigma^{q_{2}}\right) A(t)\right) \quad \forall \sigma, t>0 .
$$

Setting

$$
\max \left(\sigma^{q_{1}}, \sigma^{q_{2}}\right)=1 / s \Leftrightarrow \sigma=\min \left(s^{-1 / q_{1}}, s^{-1 / q_{2}}\right)
$$

we have

$$
\begin{array}{cc}
\min \left(s^{-1 / q_{1}}, s^{-1 / q_{2}}\right) t \leq c_{2} A^{-1}\left(\frac{A(t)}{s}\right) & \forall s, t>0 \\
\frac{t}{A^{-1}\left(\frac{A(t)}{s}\right)} \leq c_{2} \max \left(s^{1 / q_{1}}, s^{1 / q_{2}}\right) & \forall s, t>0
\end{array}
$$

thus, using (2.2),

$$
A\left(\frac{t}{A^{-1}\left(\frac{A(t)}{s}\right)}\right) \leq \tilde{c}_{2} \max \left(A\left(s^{1 / q_{1}}\right), A\left(s^{1 / q_{2}}\right)\right) \quad \forall s, t>0
$$

from which the assertion follows.

Remark 4.2. Note that $\Psi_{A}$ in (4.1) is an increasing function, such that $\Psi(0+)=0$. In the following we will use its natural extension in 0 , by setting $\Psi(0)=0$.

Let us now denote by $B_{n}(x), n \in \mathbf{Z}$, the open ball with radius $2^{-n}$ centered at $x$, and by $B_{n}$ the ball $B_{n}(0)$. We will call $\eta$ the characteristic function for $B_{0}: \eta=\chi_{B_{0}}$ so that Supp $\eta=\bar{B}_{0}, \eta$ is nonnegative, bounded, lower semicontinuous and $\eta(r x)$ is a decreasing function of $r>0$ for any $x \in \mathbf{R}^{N}$. We define $\eta_{n}, n \in \mathbf{Z}$, by setting $\eta_{n}(x)=2^{n N} \eta\left(2^{n} x\right)$ so that Supp $\eta_{n}=\bar{B}_{n}, \int \eta_{n} \mathrm{~d} x=\int \eta \mathrm{d} x$.

The main tool that will be used in the following is the generalization of the so-called Wolff's inequality (see [1], Th. 4.5.2, p. 109) in the framework of Orlicz spaces. Even if in fact the proof is a generalization of that one given in [1], we show extensively the argument, because we think that in this case the refinement of the estimations is not completely standard.

Theorem 4.3. Let $0<\alpha<N$ and $\mu \in \mathcal{M}^{+}(K)$. The following inequality holds:

$$
A\left(\left\|G_{\alpha} * \mu\right\|_{A}\right) \leq c \Psi_{A}\left(\int_{\mathbf{R}^{N}} W_{\alpha, \tilde{A}}^{\mu}(x) \mathrm{d} \mu\right)
$$

where

$$
W_{\alpha, \tilde{A}}^{\mu}(x)=\int_{0}^{4} t^{\alpha} A^{\prime}\left(\frac{\mu(B(x, t))}{t^{N-\alpha}}\right) \frac{\mathrm{d} t}{t}
$$

for some constant c depending on $A, \alpha, N$ but independent of $\mu$. 
Proof. According to Theorem 1 of [3], there exists a positive constant $c$ such that

$$
\left\|G_{\alpha} * \mu\right\|_{A} \leq c\left\|M_{\alpha, \delta} \mu\right\|_{A} \quad \forall \mu \in \mathcal{M}^{+}(K)
$$

thus

$$
A\left(\left\|G_{\alpha} * \mu\right\|_{A}\right) \leq c A\left(\left\|M_{\alpha, 1} \mu\right\|_{A}\right)
$$

and therefore, by (4.1),

$$
A\left(\left\|G_{\alpha} * \mu\right\|_{A}\right) \leq c \Psi_{A}\left(\int_{\mathbf{R}^{N}} A\left(\left|M_{\alpha, 1} \mu\right|\right) \mathrm{d} x\right) .
$$

Moreover

$$
\begin{aligned}
M_{\alpha, 1} \mu(x) & =\sup _{0<r \leq 1} \frac{\mu(B(x, r))}{|B(x, r)|^{(N-\alpha) / N}}=\sup _{n \geq 0} \sup _{2^{-n-1} \leq r \leq 2^{-n}} \frac{\mu(B(x, r))}{|B(x, r)|^{(N-\alpha) / N}} \\
& \leq \sup _{n \geq 0} \frac{\mu\left(B\left(x, 2^{-n}\right)\right)}{\left|B\left(x, 2^{-n-1}\right)\right|^{(N-\alpha) / N}}=\sup _{n \geq 0} \frac{\mu\left(B_{n}(x)\right)}{\left[c_{N}\left(2^{-n-1}\right)^{N}\right]^{(N-\alpha) / N}} \\
& =c \sup _{n \geq 0} 2^{n(N-\alpha)} \mu\left(B_{n}(x)\right)=c \sup _{n \geq 0}\left(2^{-n \alpha} \eta_{n} * \mu(x)\right) .
\end{aligned}
$$

Thus we get

$$
A\left(\left\|G_{\alpha} * \mu\right\|_{A}\right) \leq c \Psi_{A}\left(\int_{\mathbf{R}^{N}} A\left(\left|\sup _{n \geq 0}\left(2^{-n \alpha}\left(\eta_{n} * \mu\right)(x)\right)\right|\right) \mathrm{d} x\right)
$$

from which

$$
\begin{aligned}
\Psi_{A}^{-1}\left(c^{-1} A\left(\left\|G_{\alpha} * \mu\right\|_{A}\right)\right) & \leq \int_{\mathbf{R}^{N}} A\left(\left|\sup _{n \geq 0}\left(2^{-n \alpha}\left(\eta_{n} * \mu\right)(x)\right)\right|\right) \mathrm{d} x \\
& \leq \int_{\mathbf{R}^{N}} \sum_{n=0}^{\infty} A\left(2^{-n \alpha}\left(\eta_{n} * \mu\right)(x)\right) \mathrm{d} x \\
& \leq c \int_{\mathbf{R}^{N}} \sum_{n=0}^{\infty} A^{\prime}\left(2^{-n \alpha}\left(\eta_{n} * \mu\right)(x)\right) 2^{-n \alpha}\left(\eta_{n} * \mu\right)(x) \mathrm{d} x .
\end{aligned}
$$

Since $\eta_{n}$ is even and by classical properties of convolution, we get

$$
\Psi_{A}^{-1}\left(c^{-1} A\left(\left\|G_{\alpha} * \mu\right\|_{A}\right)\right) \leq c \int_{\mathbf{R}^{N}} \sum_{n=0}^{\infty} 2^{-n \alpha} A^{\prime}\left(2^{-n \alpha}\left(\eta_{n} * \mu\right)\right) * \eta_{n} \mathrm{~d} \mu
$$

and since

$$
\begin{aligned}
\eta_{n} * \mu(x) & =\int_{\mathbf{R}^{N}} \eta_{n}(x-y) \mathrm{d} \mu(y)=\int_{\mathbf{R}^{N}} 2^{n N} \eta\left(2^{n}(x-y)\right) \mathrm{d} \mu(y) \\
& =\int_{B_{n}(x)} 2^{n N} \eta\left(2^{n}(x-y)\right) \mathrm{d} \mu(y)=2^{n N} \int_{B_{n}(x)} \mathrm{d} \mu(y) \\
& =2^{n N} \mu\left(B_{n}(x)\right),
\end{aligned}
$$

one gets

$$
\eta_{n} * A^{\prime}\left(2^{-n \alpha}\left(\eta_{n} * \mu\right)\right)(x)=\int_{\mathbf{R}^{N}} \eta_{n}(x-y) A^{\prime}\left(2^{-n \alpha} 2^{n N} \mu\left(B_{n}(y)\right)\right) \mathrm{d} y .
$$


Since the last integral is in fact over $B_{n}(x)$ and $B_{n}(y) \subset B_{n-1}(x)$ for all $y \in B_{n}(x)$, we get

$$
\begin{aligned}
\eta_{n} * A^{\prime}\left(2^{-n \alpha} \eta_{n} * \mu\right)(x) & \leq A^{\prime}\left(2^{-n \alpha} 2^{n N} \mu\left(B_{n-1}(x)\right)\right) \int_{\mathbf{R}^{N}} \eta_{n}(x-y) \mathrm{d} y . \\
& =A^{\prime}\left(2^{-n \alpha} 2^{n N} \mu\left(B_{n-1}(x)\right)\right) .
\end{aligned}
$$

Thus

$$
\Psi_{A}^{-1}\left(c^{-1} A\left(\left\|G_{\alpha} * \mu\right\|_{A}\right)\right) \leq c \int_{\mathbf{R}^{N}} \sum_{n=0}^{\infty} 2^{-n \alpha} A^{\prime}\left(2^{n(N-\alpha)} \mu\left(B_{n-1}(x)\right)\right) \mathrm{d} \mu .
$$

On the other hand, setting

$$
W_{\alpha, \tilde{A}}^{\mu}(x)=\int_{0}^{4} t^{\alpha} A^{\prime}\left(\frac{\mu(B(x, t))}{t^{N-\alpha}}\right) \frac{\mathrm{d} t}{t}
$$

we have

$$
\begin{aligned}
W_{\alpha, \tilde{A}}^{\mu}(x) & =\sum_{n=0}^{\infty} \int_{2^{-n+1}}^{2^{-n+2}} t^{\alpha} A^{\prime}\left(\frac{\mu(B(x, t))}{t^{N-\alpha}}\right) \frac{\mathrm{d} t}{t} \\
& \geq \sum_{n=0}^{\infty} \int_{2^{-n+1}}^{2^{-n+2}} 2^{-n \alpha+\alpha} A^{\prime}\left(\frac{\mu\left(B\left(x, 2^{-(n-1)}\right)\right)}{2^{-n(N-\alpha)+2(N-\alpha)}}\right) \frac{\mathrm{d} t}{2^{-n+2}} \\
& =\frac{1}{2} \sum_{n=0}^{\infty} \int_{2^{-n+1}}^{2^{-n+2}} 2^{\alpha} \cdot 2^{-n \alpha} A^{\prime}\left(2^{-2(N-\alpha)} \cdot 2^{n(N-\alpha)} \mu\left(B_{n-1}(x)\right)\right) \frac{\mathrm{d} t}{2^{-n+1}} \\
& =2^{\alpha-1} \sum_{n=0}^{\infty} 2^{-n \alpha} A^{\prime}\left(2^{-2(N-\alpha)} \cdot 2^{n(N-\alpha)} \mu\left(B_{n-1}(x)\right)\right) \\
& \geq 2^{\alpha-1} c_{A^{\prime}, N, \alpha} \sum_{n=0}^{\infty} 2^{-n \alpha} A^{\prime}\left(2^{n(N-\alpha)} \mu\left(B_{n-1}(x)\right)\right) .
\end{aligned}
$$

From the relations obtained, the assertion follows.

In order to fix some more notation, let us recall the definition of Hausdorff measure. Let $h(r)$ be an increasing function, defined $(\leq+\infty)$ for $r \geq 0$, and satisfying $h(0)=0$. Let $E \subset \mathbf{R}^{N}$, and consider coverings of $E$ by countable unions of (open or closed) balls $\left\{B\left(x_{i}, r_{i}\right)\right\}_{i=1}^{\infty}$ with radii $\left\{r_{i}\right\}_{i=1}^{\infty}$. Then for any $\rho, 0<\rho \leq \infty$, a set function $\Lambda_{h}^{(\rho)}$ is defined by

$$
\Lambda_{h}^{(\rho)}(E)=\inf \sum_{i=1}^{\infty} h\left(r_{i}\right)
$$

where the infimum is taken over all such coverings with $\sup _{i=1} r_{i} \leq \rho$. Clearly $\Lambda_{h}^{(\rho)}(E)$ is a decreasing function of $\rho$, so $\lim _{\rho \rightarrow 0} \Lambda_{h}^{(\rho)}(E)$ exists $(\leq+\infty)$, and we can define

$$
\Lambda_{h}(E)=\lim _{\rho \rightarrow 0} \Lambda_{h}^{(\rho)}(E) .
$$

This is the Hausdorff measure of $E$ with respect to the function $h$. If $h(r)=r^{\alpha}$, we write $\Lambda_{\alpha}$ for $\Lambda_{r^{\alpha}}$. The set function $\Lambda_{h}^{(\infty)}$ is called the Hausdorff capacity.

Following [4], let us now give the following definition:

Definition 4.4. Let $E$ be any subset of $\mathbf{R}^{N}$. We set

$$
C_{\alpha, A}^{\prime}(E)=\inf \left\{\|f\|_{A}: f \in L^{A}\left(\mathbf{R}^{N}\right), G_{\alpha} * f \geq \chi_{E}\right\} .
$$


We have now all the background in order to prove the following:

Theorem 4.5. Let $0<\alpha<N, h$ be an increasing function on $[0, \infty[$ such that $h(0)=0$,

$$
\int_{0}^{4} t^{\alpha-1}(\widetilde{A})^{\prime}\left(\frac{h(t)}{t^{N-\alpha}}\right) \mathrm{d} t=H<\infty
$$

and let $E \subset \mathbf{R}^{N}$ be a set satisfying $\Lambda_{h}^{(\infty)}(E)>0$.

Then there exists a constant $c_{A}>0$, independent of $h$ and $E$, such that

$$
\Lambda_{h}^{(\infty)}(E) \leq \Theta\left(c_{A} C^{\prime}{ }_{\alpha, A}(E)\right)
$$

where $\Theta(t)$ is an increasing function such that $\Theta(0+)=0$. In particular,

$$
C^{\prime}{ }_{\alpha, A}(E)=0 \Rightarrow \Lambda_{h}(E)=0
$$

Proof. Let $K$ be compact with $\Lambda_{h}^{(\infty)}(K)>0$, and let $\mu \in \mathcal{M}^{+}(K)$ be given by a theorem of Frostman (see [1]), such that there is a constant $c>0$ depending only on $N$,

$$
\mu(B(x, t)) \leq h(t) \text { for all balls } B(x, t)
$$

and

$$
c^{-1} \Lambda_{h}^{(\infty)}(K) \leq \mu(K) \leq \Lambda_{h}^{(\infty)}(K)
$$

By Wolff's inequality (Th. 4.3)

$$
\begin{aligned}
\widetilde{A}\left(\left\|G_{\alpha} * \mu\right\|_{\widetilde{A}}\right) & \leq c \Psi_{\widetilde{A}}\left(\int_{\mathbf{R}^{N}} W_{\alpha, A}^{\mu}(x) \mathrm{d} \mu\right) \\
& =c \Psi_{\widetilde{A}}\left(\int_{\mathbf{R}^{N}}\left(\int_{0}^{4} t^{\alpha-1}(\widetilde{A})^{\prime}\left(\frac{\mu(B(x, t))}{t^{N-\alpha}}\right) \mathrm{d} t\right) \mathrm{d} \mu\right)
\end{aligned}
$$

and therefore, by (4.5),

$$
\begin{aligned}
\widetilde{A}\left(\left\|G_{\alpha} * \mu\right\|_{\widetilde{A}}\right) & \leq c \Psi_{\widetilde{A}}\left(\int_{\mathbf{R}^{N}}\left(\int_{0}^{4} t^{\alpha-1}(\widetilde{A})^{\prime}\left(\frac{h(t)}{t^{N-\alpha}}\right) \mathrm{d} t\right) \mathrm{d} \mu\right) \\
& =c \Psi_{\widetilde{A}}\left(\int_{\mathbf{R}^{N}} H \mathrm{~d} \mu\right) \\
& =c \Psi_{\widetilde{A}}(H \mu(K))
\end{aligned}
$$

thus

$$
\left\|G_{\alpha} * \mu\right\|_{\widetilde{A}} \leq \widetilde{A}^{-1}\left(c \Psi_{\widetilde{A}}(H \mu(K))\right)
$$

According to Theorem 11 (Part 2) of [4] (see also [2]) one has $C_{\alpha, A}^{\prime}(K)=\sup \left\{\mu(K): \mu \in \mathcal{M}^{+}(K), \mu\right.$ concentrated on $\left.K,\left\|G_{\alpha} * \mu\right\|_{\widetilde{A}} \leq 1\right\}$. As a consequence, we have

$$
C_{\alpha, A}^{\prime}(K) \geq \frac{\mu(K)}{\left\|G_{\alpha} * \mu\right\|_{\widetilde{A}}}
$$


and therefore, by (4.7),

$$
C_{\alpha, A}^{\prime}(K) \geq \frac{\mu(K)}{\widetilde{A}^{-1}\left(c \Psi_{\widetilde{A}}(H \mu(K))\right)}
$$

and by (4.6) and Remark 4.2,

$$
C^{\prime}{ }_{\alpha, A}(K) \geq c_{\widetilde{A}^{-1}} \frac{\Lambda_{h}^{(\infty)}(K)}{\widetilde{A}^{-1}\left(\Psi_{\widetilde{A}}\left(H \Lambda_{h}^{(\infty)}(K)\right)\right)}=c_{\widetilde{A}^{-1}} \Theta^{-1}\left(\Lambda_{h}^{(\infty)}(K)\right)
$$

from which the assertion follows. The property $\Theta(0+)=0$ is true because $\Theta^{-1}(t)=t / \widetilde{A}^{-1}\left(\Psi_{\widetilde{A}}(H t)\right)$ and therefore, from (4.2), we get (for $t$ small)

$$
\frac{t}{(H t)^{1 / q_{2}}} \leq \Theta^{-1}(t) \leq \frac{t}{(H t)^{1 / q_{1}}}
$$

from which

$$
H^{1 /\left(q_{1}-1\right)} t^{q_{1} /\left(q_{1}-1\right)} \leq \Theta(t) \leq H^{1 /\left(q_{2}-1\right)} t^{q_{2} /\left(q_{2}-1\right)} .
$$

The last part follows from the fact that for a general set $E, \Lambda_{h}^{(\infty)}(E)=0$ if and only if $\Lambda_{h}(E)=0$ (see [1]). Let us now extend the results to a general set $E$, not necessarily compact. There exists $E^{\prime}$ countable intersection of open sets such that $E \subset E^{\prime}$ and $\Lambda_{h}^{(\infty)}(E)=\Lambda_{h}^{(\infty)}\left(E^{\prime}\right)$ and $C^{\prime}{ }_{\alpha, A}(E)=C^{\prime}{ }_{\alpha, A}\left(E^{\prime}\right)\left(C^{\prime}{ }_{\alpha, A}\right.$ is an outer capacity according to [4], and $\Lambda_{h}^{(\infty)}$ is an outer measure according to [26]). Using the fact that $\Lambda_{h}^{(\infty)}$ satisfies the assumptions of Choquet's theorem (see [26], Chap. 2.7), one has

$$
\Lambda_{h}^{(\infty)}\left(E^{\prime}\right)=\sup \left\{\Lambda_{h}^{(\infty)}(K), K \text { compact, } K \subset E^{\prime}\right\}
$$

Moreover, using Theorem 9 of [4], one has also

$$
C^{\prime}{ }_{\alpha, A}\left(E^{\prime}\right)=\sup \left\{\mathrm{C}^{\prime}{ }_{\alpha, A}(K), K \text { compact, } K \subset E^{\prime}\right\} .
$$

Hence the results obtained for compact sets can be extended to general sets.

Remark 4.6. The proof of Theorem 4.5 follows the ideas used to prove the Theorem 5.1.13 of [1] (p. 137). We remark that, with respect to the original proof, our constant is rougher, but simpler, and the proof is slightly shorther. In our context this (small) simplification is possible because we do not need finer constants.

Let us now consider some relations between Definition 2.5, Definition 2.6 and the Definition 4.4. Let us first consider the case $A(t)=t^{r}, r>1$ : Definition 4.4 reduces to

$$
C_{\alpha, r}^{\prime}(E)=\inf \left\{\|f\|_{r}: f \in L^{r}\left(\mathbf{R}^{N}\right), G_{\alpha} * f \geq \chi_{E}\right\} .
$$

Denoting by $L^{\alpha, r}\left(\mathbf{R}^{N}\right)$ the Bessel potential spaces

$$
L^{\alpha, r}\left(\mathbf{R}^{N}\right)=\left\{h: h=G_{\alpha} * f, f \in L^{r}\left(\mathbf{R}^{N}\right)\right\},
$$

whose norm is given by $\|h\|_{\alpha, r}=\|f\|_{r}$, we can write also

$$
C_{\alpha, r}^{\prime}(E)=\inf \left\{\|h\|_{\alpha, r}: h \in L^{\alpha, r}\left(\mathbf{R}^{N}\right), h \geq \chi_{E}\right\} .
$$


At this point we use the result of Calderon: for $\alpha \in \mathbf{N}, W^{\alpha, r}\left(\mathbf{R}^{N}\right)=L^{\alpha, r}\left(\mathbf{R}^{N}\right), 1<r<\infty$, with equivalence of norms, i.e. there is a constant $c$ such that for all $f$

$$
c^{-1}\|f\|_{\alpha, r} \leq\|f\|_{W^{\alpha, r}} \leq c\|f\|_{\alpha, r} .
$$

Hence it is clear that $C_{\alpha, r}^{\prime}$ is equivalent to

$$
\operatorname{cap}_{\alpha, r}^{1 / r}\left(E, \mathbf{R}^{N}\right)=\inf \left\{\|h\|_{W^{\alpha, r}}: h \in C_{c}^{\infty}\left(\mathbf{R}^{N}\right), h \geq \chi_{E}\right\} .
$$

Similarly, one can prove that the capacity $C_{1, A}^{\prime}$ defined in Definition 4.4 is equivalent to $A^{-1}\left(\operatorname{cap}_{1, A}\left(E, \mathbf{R}^{N}\right)\right)$ where

$$
\operatorname{cap}_{1, A}\left(E, \mathbf{R}^{N}\right)=\inf \left\{A\left(\|\nabla h\|_{A}+\|h\|_{A}\right): h \in C_{c}^{\infty}\left(\mathbf{R}^{N}\right), h \geq \chi_{E}\right\} .
$$

This extension is possible because the proof of Calderon's theorem can be obtained in the context of Orlicz spaces by straightforward generalization of that one given in [27] (see Chap.V, Sect. 3, Th. 3). All the properties of Orlicz spaces needed in the proof are heredited from those ones true for Lebesgue spaces. We briefly list them and give references for their proofs. For separability properties (density of $C_{0}^{\infty}\left(\mathbf{R}^{N}\right)$ in $L^{A}\left(\mathbf{R}^{N}\right)$ and in $\left.W^{1, A}\left(\mathbf{R}^{N}\right)\right)$ we refer to Section 2 of [12]. For properties obtained by interpolation, we refer to the paper [15]. Finally, for the boundedness of the Riesz transforms $R_{j}$ in Orlicz spaces we refer to the book by Kokilashvili and Krbec [16] (Th. 3.1.1, p. 97).

Proof of Theorem 2.21. Let $E$ be such that $\operatorname{cap}_{1, A}(E)=0$. Let us consider $K$ a compact set, let $\varphi_{n} \in$ $C_{c}^{\infty}(\Omega)$ such that $\varphi_{n} \geq \chi_{K}$ and $A\left(\left\|\nabla \varphi_{n}\right\|_{A}\right) \leq \operatorname{cap}_{1, A}(K)+\frac{1}{n}$. Using Poincaré inequality on $\Omega$, we have $A\left(\left\|\nabla \varphi_{n}\right\|_{A}+\left\|\varphi_{n}\right\|_{A}\right) \leq c\left(\operatorname{cap}_{1, A}(K)+\frac{1}{n}\right)$. Moreover such $\varphi_{n}$ can be used in the definition of $\operatorname{cap}_{1, A}\left(K, \mathbf{R}^{N}\right)$, thus $\operatorname{cap}_{1, A}\left(K, \mathbf{R}^{N}\right) \leq c\left(\operatorname{cap}_{1, A}(K)+\frac{1}{n}\right)$ that is $\operatorname{cap}_{1, A}\left(K, \mathbf{R}^{N}\right) \leq c \operatorname{cap}_{1, A}(K)$. And, thanks to the equivalence of capacities written above, we have $A\left(C_{1, A}^{\prime}(K)\right) \leq c \operatorname{cap}_{1, A}(K)$. Using now Theorem 2 and Theorem 9 of [4], one has for all open sets $U$

$$
C_{1, A}^{\prime}(U)=\sup \left\{C_{1, A}^{\prime}(K), K \text { compact }, K \subset U\right\},
$$

and for all set $B$

$$
C_{1, A}^{\prime}(B)=\inf \left\{C_{1, A}^{\prime}(U), U \text { open, } B \subset U\right\} .
$$

Hence $A\left(C_{1, A}^{\prime}(B)\right) \leq c \operatorname{cap}_{1, A}(B)$, for all Borelian $B$, thus $C^{\prime}{ }_{1, A}(E)=0$.

According to $(2.24), h(t)=t^{N-\beta s}$ satisfies the hypotheses of Theorem 4.5 with $\alpha=1$, thus $\Lambda_{h}(E)=0$. Using now Theorem 5.1 .9 (p. 134 in [1]), we get $\operatorname{cap}_{\beta, s}\left(E, \mathbf{R}^{N}\right)=0$. It remains now to prove that $\operatorname{cap}_{\beta, s}(E)=$ $\operatorname{cap}_{\beta, s}(E, \Omega)=0$.

Since $\operatorname{cap}_{\beta, s}\left(E, \mathbf{R}^{N}\right)=0$, there exists a sequence $U_{n}$ of open sets in $\mathbf{R}^{N}$ such that $E \subset U_{n}$ and $\operatorname{cap}_{\beta, s}\left(U_{n}, \mathbf{R}^{N}\right) \leq \frac{1}{n}$. Since the capacity is nondecreasing, we can suppose that $U_{n} \subset \Omega$ (by replacing $U_{n}$ with $U_{n} \cap \Omega$, recall that $\left.E \subset \Omega\right)$. Hence there exists $h_{n} \in C_{c}^{\infty}\left(\mathbf{R}^{N}\right)$ such that $h \geq_{U_{n}}$ and $\left\|h_{n}\right\|_{W^{\beta, s}\left(\mathbf{R}^{N}\right)}^{s} \leq \frac{2}{n}$. Let now be $\widetilde{K}$ a compact set in $\Omega$, and $\widetilde{U}$ an open set such that $\widetilde{K} \subset \widetilde{U} \subset \subset \Omega$. There exists $\xi \in C_{c}^{\infty}(\Omega)$ such that $\xi \geq \chi_{\widetilde{U}}$. Hence $\xi h_{n} \in C_{c}^{\infty}(\Omega), \xi h_{n} \geq \chi_{\tilde{U} \cap U_{n}}$ and $\left\|\xi h_{n}\right\|_{W^{\beta, s}}^{s} \leq c\left\|h_{n}\right\|_{W^{\beta, s}}^{s} \leq \frac{c}{n}$. Thus for all $K \subset \widetilde{U} \cap U_{n}$, $\operatorname{cap}_{\beta, s}(K, \Omega) \leq \frac{c}{n}$, hence $\operatorname{cap}_{\beta, s}\left(\widetilde{U} \cap U_{n}, \Omega\right) \leq \frac{c}{n}$. Finally, since $E \cap \widetilde{K} \subset \widetilde{U} \cap U_{n}, \operatorname{cap}_{\beta, s}(E \cap \widetilde{K}, \Omega) \leq \frac{c}{n}$ for all $n \in N$ so $\operatorname{cap}_{\beta, s}(E \cap \widetilde{K})=0$. Since $\Omega$ is the union of increasing compact sets, one has $\operatorname{cap}_{\beta, s}(E)=\operatorname{cap}_{\beta, s}(E, \Omega)=0$.

This paper has been written while the first author was visiting the Laboratoire d'Analyse Numérique, Université Paris VI, invited by François Murat. He thanks for the warm hospitality.

Support. The first author has been supported by Italian M.U.R.S.T. research funds. 


\section{REFERENCES}

[1] D.R. Adams and L.I. Hedberg, Function spaces and potential theory. Springer-Verlag, Berlin, Grundlehren Math. Wiss. 314 (1996).

[2] N. Aissaoui, Bessel potentials in Orlicz spaces. Rev. Mat. Univ. Complut. Madrid 10 (1997) 55-79.

[3] N. Aissaoui, Some developments of Strongly Nonlinear Potential Theory. Libertas Math. 19 (1999) 155-170.

[4] N. Aissaoui and A. Benkirane, Capacités dans les espaces d'Orlicz. Ann. Sci. Math. Québec 18 (1994) 1-23.

[5] P. Baras and M. Pierre, Singularités éliminables pour des équations semi-linéaires. Ann. Inst. Fourier (Grenoble) 34 (1984) 185-206.

[6] P. Bénilan, L. Boccardo, T. Gallouët, R. Gariepy, M. Pierre and J.L. Vazquez, An $L^{1}$ theory of existence and uniqueness of nonlinear elliptic equations. Ann. Scuola Norm. Sup. Pisa Cl. Sci. 22 (1995) 240-273.

[7] P. Bénilan, H. Brezis and M. Crandall, A semilinear elliptic equation in $L^{1}\left(\mathbf{R}^{N}\right)$. Ann. Scuola Norm. Sup. Pisa Cl. Sci. 2 (1975) 523-555.

[8] L. Boccardo and T. Gallouët, Nonlinear elliptic equations with right-hand side measures. Comm. Partial Differential Equations 17 (1992) 641-655.

[9] H. Brezis, Nonlinear elliptic equations involving measures, in Contributions to nonlinear partial differential equations (Madrid, 1981). Pitman, Boston, Mass.-London, Res. Notes in Math. 89 1983) 82-89.

[10] G. Choquet, Theory of Capacities, Ann. Inst. Fourier (Grenoble) 5 (1953-1954) 131-295 (Ch. 1, Thm 4.1, p. 142).

[11] G. Dal Maso, F. Murat, L. Orsina and A. Prignet, Renormalized solutions for elliptic equations with general measure data. Ann. Scuola Norm. Sup. Pisa CL. Sci. 28 (1999) 741-808.

[12] T.K. Donaldson and N.S. Trudinger, Orlicz-Sobolev spaces and embedding theorems. J. Funct. Anal. 8 (1971) 52-75.

[13] A. Fiorenza, An inequality for Jensen Means. Nonlinear Anal. 16 (1991) 191-198.

[14] T. Gallouët and J.M. Morel, Resolution of a semilinear equation in L $L^{1}$. Proc. Roy. Soc. Edinburgh 96 (1984) 275-288.

[15] J. Gustavsson and J. Peetre, Interpolation of Orlicz spaces. Studia Math. 60 (1977) 33-59.

[16] V. Kokilashvili and M. Krbec, Weighted inequalities in Lorentz and Orlicz spaces. World Scientific (1991).

[17] M.A. Krasnosel'skii and Ya.B. Rutickii, Convex functions and Orlicz Spaces. Noordhoff Ltd. (1961).

[18] J. Leray and J.-L. Lions, Quelques résultats de Višik sur les problèmes elliptiques non linéaires par les méthodes de MintyBrowder. Bull. Soc. Math. France 93 (1965) 97-107.

[19] L. Maligranda, Orlicz Spaces and Interpolation. Dep. de Matematica Univ. Estadual de Campinas, Campinas, Brazil (1989).

[20] J. Malý, Coarea properties of Sobolev functions, in Proc. Function Spaces, Differential Operators and Nonlinear Analysis (The Hans Triebel Anniversary Volume). Birkhäuser, Basel (to appear).

[21] J. Malý, D. Swanson and W.P. Ziemer, Fine behavior of functions with gradient in a Lorentz space (in preparation).

[22] V.G. Maz'ja and V.P. Havin, Nonlinear potential theory. Uspekhi Mat. Nauk 27 (1972) 67-138. English translation: Russian Math. Surveys 27 (1972) 71-148.

[23] L. Orsina and A. Prignet, Nonexistence of solutions for some nonlinear elliptic equations involving measures. Proc. Roy. Soc. Edinburgh Ser. A 130 (2000) 167-187.

[24] L.E. Persson, Interpolation with a parameter function. Math. Scand. 59 (1986) 199-222.

[25] M.M. Rao and Z.D. Ren, Theory of Orlicz Spaces. Marcel Dekker (1991).

[26] C.A. Rogers, Hausdorff Measures. Cambridge University Press (1970).

$[27]$ E.M. Stein, Singular Integrals and Differentiability properties of functions. Princeton University Press (1970). 